Pacific

Journal of

Mathematics

GROUPS THAT ACT PSEUDOFREELY ON $S^{2} \times S^{2}$

Michael P. McCoOeY

Volume $230 \quad$ No. 2

April 2007 


\title{
GROUPS THAT ACT PSEUDOFREELY ON $S^{2} \times S^{2}$
}

\author{
Michael P. McCoOEY
}

\begin{abstract}
A pseudofree group action on a space $X$ is one whose set of singular orbits forms a discrete subset of its orbit space. Equivalently - when $G$ is finite and $X$ is compact - the set of singular points in $X$ is finite. In this paper, we classify all of the finite groups which admit pseudofree actions on $S^{2} \times S^{2}$. The groups are exactly those that admit orthogonal pseudofree actions on $S^{2} \times S^{2} \subset \mathbb{R}^{3} \times \mathbb{R}^{3}$, and they are explicitly listed.

This paper can be viewed as a companion to a preprint of Edmonds, which uniformly treats the case in which the second Betti number of a fourmanifold $M$ is at least three.
\end{abstract}

\section{Introduction}

The classification of free actions of groups on spheres and products of spheres is an important problem in topology. In even dimensions, however, the question of which groups admit free actions is much less interesting: It follows from the Lefschetz fixed point theorem that such a group must admit a faithful, and rather special, representation on homology. Thus, for example, $\mathbb{Z}_{2}$ is the only group which can act freely on $S^{2 n}$, or freely and orientably on $S^{2 n} \times S^{2 n}$. The Lefschetz fixed point theorem puts the same sort of restriction on free actions of groups on closed, simply-connected four-manifolds: in the homologically trivial case, there are none. This is one motivation for the concept of pseudofree actions.

An action of a finite group $G$ on a space $X$ is pseudofree if it is free on the complement of a discrete set of points. (The use of this term is not consistent across the literature. Some authors also require that each singular point be fixed by the entire group.) Such actions make the singular set of the group action as small as is compatible with the Lefschetz theorem. It is a theorem of Edmonds [1997a] that if $b_{2}\left(M^{4}\right) \geq 3$, the only groups which can act pseudofreely on $M$ and trivially on $H_{*}(M)$ are cyclic. In this paper, we treat the case $M=S^{2} \times S^{2}-$ which is especially interesting in its own right — for all possible actions on homology.

MSC2000: primary 55M35, 57S25, 57S17; secondary 20J06, 55T10.

Keywords: pseudofree, group, group action, four-manifold. 
Let $G$ be a finite group with an action on $S^{2} \times S^{2}$ which preserves orientation and is locally linear and pseudofree. Two questions arise naturally: Which groups $G$ admit such actions? And given a group which acts pseudofreely, can we classify the actions? A partial answer to the latter question is known: if $G$ is cyclic, the Wilczyński-Bauer invariants [Bauer and Wilczyński 1996; Wilczyński 1994], detect whether two given pseudofree actions (on any simply-connected fourmanifold) are topologically conjugate. The work of Edmonds and Ewing [1992], as well as that of other authors, addresses the question of which combinations of these invariants are realizable, but there are number-theoretical difficulties involved in making the answer explicit.

Evidence from the study of other simple 4-manifolds (see [Cappell and Shaneson 1979; Kulkarni 1982] for the case of $S^{4}$ and [Wilczyński 1987; Hambleton and Lee 1988] for $\mathbb{C} P^{2}$ ) hints at an answer to the first question: the groups which admit pseudofree actions are exactly those which can also act pseudofreely and orthogonally on $S^{2} \times S^{2} \subset \mathbb{R}^{6}$. Our main result is that this is indeed the case: we classify the groups acting orthogonally and pseudofreely, and prove that these are the only groups admitting even locally linear pseudofree actions. (More precisely, the groups and their corresponding representations on homology are exactly those which occur in the linear case.) The remaining problem, that of classifying the actions themselves, is left for future work.

Here is a brief outline of the paper: The homology representation of a group acting on $S^{2} \times S^{2}$ gives rise to a short exact sequence. Certain group extension problems related to this sequence are solved, and then the geometry of linear actions is used to determine which of these extensions admit pseudofree orthogonal actions. Actions are constructed in the course of the proofs. The classification of groups acting linearly and pseudofreely is stated explicitly in Theorem 3.9, and is reformulated in terms of the homology representation in Corollary 3.10. In Section 4, the conditions of Corollary 3.10 are shown to be necessary in the locally linear case, as well, thereby proving the main theorem. The proof uses a series of lemmas which apply orbit-counting arguments to the singular set of a group action, and then some group-theoretical and cohomological calculations to rule out minimal potential pathologies.

\section{Group theory}

Suppose a group $G$ acts on $S^{2} \times S^{2}$. The induced action of $G$ on $H_{2}\left(S^{2} \times S^{2}\right)$ defines a representation $\varphi: G \rightarrow G L(2, \mathbb{Z})$. Since $\varphi$ must respect the intersection form, it must leave the positive and negative definite subspaces of $\mathrm{H}_{2}\left(\mathrm{~S}^{2} \times S^{2}\right)$ invariant. $H_{2}^{+}$is spanned (rationally) by $x+y$, and $H_{2}^{-}$is spanned by $x-y$, where $x$ and $y$ represent the standard generators. With respect to this basis, $\varphi(g)$ must have the 
form $\left(\begin{array}{cc} \pm 1 & 0 \\ 0 & \pm 1\end{array}\right)$ for any $g \in G$. It follows that with respect to the standard basis,

$$
\varphi(g) \in\left\langle \pm\left(\begin{array}{ll}
1 & 0 \\
0 & 1
\end{array}\right), \pm\left(\begin{array}{ll}
0 & 1 \\
1 & 0
\end{array}\right)\right) \cong \mathbb{Z}_{2} \times \mathbb{Z}_{2} .
$$

Thus the representation on homology induces a short exact sequence

$$
1 \rightarrow K \rightarrow G \stackrel{\varphi}{\rightarrow} Q \rightarrow 1,
$$

where $K$ acts trivially on homology and $Q \subset \mathbb{Z}_{2} \times \mathbb{Z}_{2}$. This sequence allows us to approach general $G$-actions by first considering homologically trivial actions and then dealing with associated extension problems. In this section, we address the group theory involved in the extension problems, and also some cohomology calculations which will be needed later on in the nonlinear case. The results, though technical, are for the most part routine.

First recall some generalities about the classification of group extensions:

If $K$ is an abelian group, and $1 \rightarrow K \rightarrow G \rightarrow Q \rightarrow 1$ is an extension, then $G$ acts on $K$ by conjugation. Since $K$ acts trivially on itself, conjugation induces a well-defined action of $Q$ on $K$. For a specific $g \in G$, the conjugation automorphism $h \mapsto g^{-1} h g$ will be denoted $\mu_{g}$. The action of $Q$ defines a homomorphism $\psi$ : $Q \rightarrow$ Aut $K$ and a $Q$-module structure on $K$. As is well known, the extensions $1 \rightarrow K \rightarrow G \rightarrow Q \rightarrow 1$ which give rise to the action $\psi$ of $Q$ on $K$ are classified by $H^{2}(Q ; K)$.

If $K$ is nonabelian, the situation is a bit more delicate: an extension problem $1 \rightarrow K \rightarrow G \rightarrow Q \rightarrow 1$ with nonabelian kernel is described by the "abstract kernel" $(K, Q, \psi)$, where $\psi: Q \rightarrow$ Out $K$ describes the "outer action" of $Q$ on $K$. For general $(K, Q, \psi)$, an extension might not exist: $\psi$ determines an obstruction cocycle in $H^{3}(Q ; Z(K))$. The obstruction measures, roughly, whether it is possible to simultaneously realize the (outer) actions of each element of $Q$ by conjugations in $G$. If the obstruction vanishes, then the extensions realizing $(K, Q, \psi)$ are in (noncanonical) one-to-one correspondence with $H^{2}(Q ; Z(K))$. For detailed accounts of this theory, see [Brown 1982] or [Mac Lane 1975].

In this section, we will mainly be concerned with extensions with quotient $\mathbb{Z}_{2}$. Assume such an extension exists, and pick some $q \in G \backslash K$. Two pieces of data determine the structure of $G$ :

(1) The particular value in $K$ of the square of $q$. Denote it by $g_{q^{2}}$.

(2) The automorphism $\mu_{q}$ of $K$. In the abstract (when a specific extension is not given in advance), this automorphism will be denoted $\gamma$.

The choices of $g_{q^{2}}$ and $\gamma$ are not arbitrary: $\gamma$ must always fix $g_{q^{2}}$, and $\gamma^{2}$ must be an inner automorphism of $K$. In specific instances, there may be other restrictions as well. 
Notation. The dihedral group of order $2 m$ will be denoted $D_{m}$. Similarly, the binary dihedral group of order $4 m$, with presentation $\langle k, q| k^{m}=q^{2}, q^{-1} k q=$ $\left.k^{-1}\right\rangle$, will be denoted $D_{m}^{*}$. (This group is also commonly referred to as a dicyclic or generalized quaternion group.)

Lemma 2.1. Let $K \cong \mathbb{Z}_{n}$. Each extension $0 \rightarrow K \rightarrow G \rightarrow \mathbb{Z}_{2} \rightarrow 1$ is of one of the following forms:

(1) $\mathbb{Z}_{n} \rtimes \mathbb{Z}_{2}$, where the semidirect product automorphism is given by a certain tuple $\left(\delta_{2}, \epsilon_{2}, \ldots, \epsilon_{p_{i}}, \ldots, \epsilon_{p_{k}}\right)$. Every split extension is of this type.

(2) $\left(\mathbb{Z}_{m_{-}} \rtimes \mathbb{Z}_{2^{n_{2}+1}}\right) \times \mathbb{Z}_{m_{+}}$, where the semidirect product automorphism is inversion.

(3) $D_{2^{n_{2}-1} m_{-}}^{*} \times \mathbb{Z}_{m_{+}}$.

If $n$ is odd, then every extension is split. Notation is explained in the proof.

Proof. We use additive notation for the group operation in $\mathbb{Z}_{n}$.

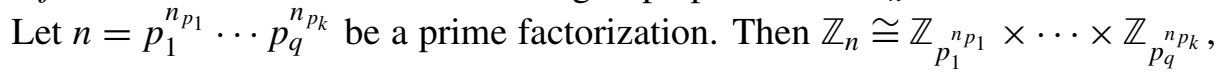
and $\gamma$ restricts to an automorphism of each of the factors.

If $p$ is odd, Aut $\mathbb{Z}_{p^{n} p}$ is cyclic, so each order two automorphism of $\mathbb{Z}_{p^{n} p}$ sends a generator to its inverse. However, Aut $\mathbb{Z}_{2^{n_{2}}} \cong \mathbb{Z}_{2} \times \mathbb{Z}_{2^{n_{2}-2}}$, so if $n_{2}>2, \mathbb{Z}_{2^{n_{2}}}$ has three automorphisms of order two: $x \mapsto-x, x \mapsto\left(1+2^{n_{2}-1}\right) x$, and $x \mapsto\left(-1+2^{n_{2}-1}\right) x$. Thus $\left.\gamma\right|_{\mathbb{Z}_{2^{n_{2}}}}$ is encoded by a pair $\left(\delta_{2}, \epsilon_{2}\right)$, where $\delta_{2}=1$ or $0, \epsilon_{2}= \pm 1$, and $\left(\delta_{2}, \epsilon_{2}\right)$ : $x \mapsto\left(\epsilon_{2}+\delta_{2} \cdot 2^{n_{2}-1}\right) x$. For $p>2$, we simply have $\left.\gamma\right|_{\mathbb{Z}_{p^{n}}}: x \mapsto \epsilon_{p} \cdot x$. With this notation, we write $\gamma=\left(\delta_{2}, \epsilon_{2}, \ldots, \epsilon_{p_{i}}, \ldots, \epsilon_{p_{k}}\right)$. Then $\mathbb{Z}_{n}$ can be viewed as a product

$$
\mathbb{Z}_{n} \cong \mathbb{Z}_{2^{n_{2}}} \times \mathbb{Z}_{m_{-}} \times \mathbb{Z}_{m_{+}},
$$

where $\mathbb{Z}_{m_{-}}$is the (odd) -1-eigenspace for $\gamma$, and $\mathbb{Z}_{m_{+}}$is the (odd) +1-eigenspace.

Since $\gamma$ fixes $g_{q^{2}}, g_{q^{2}} \equiv 0\left(\bmod p^{n_{p}}\right)$ for each odd $p$ with $\epsilon_{p}=-1$, so $g_{q^{2}} \equiv$ $0\left(\bmod m_{-}\right)$. On the other hand, any element of $\mathbb{Z}_{m_{+}}$is a multiple of 2 , so $q$ may be normalized to be trivial $\bmod m_{+}$, as well. We may therefore assume that $g_{q^{2}} \in \mathbb{Z}_{2^{n_{2}}} \times 0 \times 0$, and we have a subextension $1 \rightarrow \mathbb{Z}_{2^{n_{2}}} \rightarrow G^{\prime} \rightarrow \mathbb{Z}_{2} \rightarrow 1$. These are classified by $H^{2}\left(\mathbb{Z}_{2} ; \mathbb{Z}_{2^{n_{2}}}\right)$, where $\left(\delta_{2}, \epsilon_{2}\right)$ defines the module structure on $\mathbb{Z}_{2^{n_{2}}}$. Brown [1982, IV.4.2] computes that if $\delta_{2}=1$, then $H^{2}\left(\mathbb{Z}_{2} ; \mathbb{Z}_{2^{n_{2}}}\right)=0$, so every extension is split. Thus if $\delta_{2}=1$, the main extension $1 \rightarrow \mathbb{Z}_{n} \rightarrow G \rightarrow$ $\mathbb{Z}_{2} \rightarrow 1$ is a semidirect product. Henceforth we assume $\delta_{2}=0$. We now have $H^{2}\left(\mathbb{Z}_{2} ; \mathbb{Z}_{2^{n_{2}}}\right) \cong \mathbb{Z}_{2}$, so $H^{2}\left(\mathbb{Z}_{2} ; \mathbb{Z}_{n}\right) \cong \mathbb{Z}_{2}$, as well. The split extensions are again semidirect products. But for each $\gamma$, there is one nonsplit extension. Since $\mathbb{Z}_{m_{+}}$is central in $G$, the sequence can be written

$$
0 \rightarrow\left(\mathbb{Z}_{2^{n_{2}}} \times \mathbb{Z}_{m_{-}}\right) \times \mathbb{Z}_{m_{+}} \rightarrow G^{\prime \prime} \times \mathbb{Z}_{m_{+}} \rightarrow \mathbb{Z}_{2} \rightarrow 1,
$$

so we may pretend for the moment that $\mathbb{Z}_{m_{+}}=\{0\}$, and focus attention on $G^{\prime \prime}$. 
The structure of $G^{\prime \prime}$ depends on the sign of $\epsilon_{2}$. If $\epsilon_{2}=1$, then $\mathbb{Z}_{2^{n_{2}}}$ is central, and $G^{\prime \prime} \cong \mathbb{Z}_{m_{-}} \rtimes \mathbb{Z}_{2^{n_{2}+1}}$. If $\epsilon_{2}=-1$, then $G^{\prime \prime} \cong D_{2^{n_{2}-1} m_{-}}^{*}$. By appending the $\mathbb{Z}_{m_{+}}$ factor, we recover $G$ in each case.

Next we consider the possibility that $K$ is a dihedral group. Since extensions of dihedral groups have nonabelian kernel, the algebra is a little more technical than in the cyclic case.

Given an automorphism $\gamma$ of $D_{n}$ whose square is inner, there exists $g_{q^{2}} \in D_{n}$ such that $\gamma^{2}=\mu_{g_{q^{2}}}$, and this choice of $g_{q^{2}}$ is unique up to a factor in $Z\left(D_{n}\right)$. $Z\left(D_{n}\right)$ is trivial if $n$ is odd, and has order 2 if $n$ is even. Clearly, $g_{q^{2}}$ is fixed by $\gamma^{2}$. Working through the definitions in [Brown 1982] or [Mac Lane 1975] shows that the obstruction to realizing the abstract kernel $\left(D_{n}, \mathbb{Z}_{2}, \gamma\right)$ as an extension vanishes exactly if $g_{q^{2}}$ is fixed by $\gamma$. It is straightforward to verify that vanishing of the obstruction depends only on the outer automorphism class of $\gamma$.

Claim 1. Aut $D_{n} \approx \begin{cases}S_{3} & \text { if } n=2, \\ \mathbb{Z}_{n} \rtimes \text { Aut } \mathbb{Z}_{n} & \text { if } n>2 .\end{cases}$

We use the presentation $D_{n} \approx\left\langle s, t \mid s^{n}=t^{2}=1, t s t=s^{-1}\right\rangle$.

If $n=2$, then explicitly checking that the 2-cycle $(s, t)$ and the 3-cycle $(s, t, s t)$ respect the group operation shows that any permutation of $\{s, t, s t\}$ defines an automorphism of $D_{2}$.

If $n>2$ then, since an automorphism must send $s$ to another element of order $n$, and $t$ to an element which does not commute with $f(s)$, any automorphism is of the form $f_{a, b}$, where $f_{a, b}(t)=s^{a} t$, and $f_{a, b}(s)=s^{b}$, for $a \in \mathbb{Z}_{n}$ and $(b, n)=1$. By calculating $f_{a, b} \circ f_{c, d}=f_{b c+a, b d}$, we see that Aut $D_{n}$ is a semidirect product, as claimed.

We first deal with the case $n=2$. Since $D_{2}$ is abelian, $\gamma^{2}=1$.

Case 1: $\gamma$ is nontrivial. For convenience, we choose generators $a, b$ of $D_{2}$, and assume that $\gamma$ transposes them, leaving $a b$ fixed. Since $g_{q^{2}}$ must be fixed by $\gamma$, $g_{q^{2}}=a b$ or 1 ; replacing $g_{q}$ by $a q$ if necessary, we may assume $q^{2}=1$. Then $G=\left\langle a, b, q \mid a^{2}=b^{2}=(a b)^{2}=q^{2}=1, q a q^{-1}=b\right\rangle$, or more simply, setting $s=a q, t=b, G=\left\langle s, t \mid s^{4}=t^{2}=1, t s t=s^{-1}\right\rangle$, with $D_{2}$ included as the subgroup $\left\langle s^{2}, t\right\rangle$.

Case 2: $\gamma$ is trivial. In this case, $G$ is abelian. It is straightforward to check that $G=\mathbb{Z}_{4} \times \mathbb{Z}_{2}$ or $G=\mathbb{Z}_{2} \times \mathbb{Z}_{2} \times \mathbb{Z}_{2}$.

Assume henceforth that $n>2$.

Claim 2. Inn $D_{n} \approx 2 \mathbb{Z}_{n} \rtimes\{ \pm 1\} \subset \mathbb{Z}_{n} \rtimes$ Aut $\mathbb{Z}_{n}$.

It suffices to check that $\mu_{s^{a}}=f_{2 a, 1}$, and $\mu_{t}=f_{0,-1}$.

Now let $\gamma=f_{a, b}$. Then $\gamma^{2}=f_{a b+a, b^{2}}$. If $\gamma^{2} \in \operatorname{Inn} D_{n}$, then $b^{2}= \pm 1$. But if $b^{2}= \pm 1$, then $b+1$ must be a multiple of 2 in $\mathbb{Z}_{n}$, so $a b+a \in 2 \mathbb{Z}_{n}$ for any $a \in \mathbb{Z}_{n}$. 
Thus $\gamma^{2}$ is inner if and only if $b^{2}= \pm 1$. Since $\gamma^{2}$ must be inner for an extension to exist, we assume henceforth that $b^{2}= \pm 1$.

Claim 3. If $n$ is odd, the obstruction always vanishes. If $n$ is even and $b^{2} \equiv 1$ $(\bmod n)$, the obstruction vanishes unless $a$ and $\left(b^{2}-1\right) / n$ are both odd in $\mathbb{Z}$. If $n$ is even and $b^{2} \equiv-1(\bmod n)$, the obstruction vanishes unless a and $\left(b^{2}+1\right) / n$ are both odd in $\mathbb{Z}$.

Suppose $n$ is even. Then the equation $2 b^{\prime}=b+1$ has two solutions $\bmod n$. Fix one. The other is $b^{\prime}+n / 2$.

Case 1: $b^{2}=1$. In this case, $\gamma^{2}=\mu_{\left(s^{\left.a b^{\prime}\right)}\right.}$, so $g_{q^{2}}=s^{a b^{\prime}}$ or $s^{a\left(b^{\prime}+n / 2\right)}$. If the first is fixed by $\gamma$, so is the second. So we ask: when is $\gamma\left(s^{a b^{\prime}}\right)=s^{a b^{\prime}}$ ?

Since $\gamma\left(s^{a b^{\prime}}\right)=s^{b a b^{\prime}}, g_{q^{2}}$ is fixed by $\gamma$ if and only if $(b-1) a b^{\prime} \equiv 0(\bmod n)$. Notice that $2(b-1) a b^{\prime}=a\left(b^{2}-1\right) \equiv 0(\bmod n)$. However, it is possible that $(b-1) a b^{\prime} \equiv n / 2(\bmod n)$. This occurs if $2(b-1) a b^{\prime}=a\left(b^{2}-1\right)$ is an odd multiple of $n$, which occurs only if $a$ and $\left(b^{2}-1\right) / n$ are both odd.

Case 2: $b^{2}=-1$. Now $\gamma^{2}=\mu_{\left(s^{a b^{\prime}} t\right)}$, and $g_{q^{2}}=s^{a b^{\prime}} t$ or $s^{a\left(b^{\prime}+n / 2\right)} t \cdot \gamma$ fixes $g_{q^{2}}=$ $s^{a b^{\prime}} t$ if and only if $a b^{\prime} \equiv b a b^{\prime}+a(\bmod n)$, if and only if $(b-1) b^{\prime} a+a \equiv 0(\bmod n)$. As above, everything works unless $2\left((b-1) b^{\prime} a+a\right)=\left(b^{2}-1\right) a+2 a=\left(b^{2}+1\right) a$ is an odd multiple of $n$. This occurs only if $a$ and $\left(b^{2}+1\right) / n$ are both odd.

If $n$ is odd, then similar but easier calculations show that $g_{q^{2}}$ is always fixed by $\gamma$. This establishes the claim.

Definition. Let us say that an automorphism $\gamma$ of $D_{n}$ is admissible if an extension $1 \rightarrow D_{n} \rightarrow G \rightarrow \mathbb{Z}_{2} \rightarrow 1$ exists with $\mu_{k}=\gamma$ for some $k \in G \backslash D_{n}$.

We have just seen that for $n>2, \gamma=f_{a, b}$ is admissible if and only if $b^{2} \equiv \pm 1$ $(\bmod n)$ and the obstruction mentioned in claim 3 vanishes.

When extensions exist, they are in correspondence with

$$
H^{2}\left(\mathbb{Z}_{2}, Z\left(D_{n}\right)\right) \approx \begin{cases}0 & \text { if } n \text { is odd } \\ \mathbb{Z}_{2} & \text { if } n>2 \text { is even }\end{cases}
$$

If $n$ is even, the extensions correspond to the two choices of $g_{q^{2}}$, which differ by the nontrivial element in $Z\left(D_{n}\right)$. Thus

$$
\begin{aligned}
G & \cong\left\langle s, t, q \mid s^{n}=t^{2}=1, t s t=s^{-1}, q^{2}=g_{q^{2}}, q^{-1} s q=\gamma(s), q^{-1} t q=\gamma(t)\right\rangle \\
& \left.\cong\left\langle D_{n}, q\right| q^{2}=g_{q^{2}}, q^{-1} g q=\gamma(g) \text { for } g \in D_{n}\right\rangle .
\end{aligned}
$$

This presentation depends on a particular $\gamma \in[\gamma] \in$ Out $D_{n}$. For some purposes, we might wish to normalize $g_{q^{2}}$. To this end, note that for $g \in D_{n}$,

$$
\left(\mu_{g} \circ \gamma\right)^{2}=\mu_{g \gamma(g)} \circ \gamma^{2}=\mu_{g \gamma(g) g_{q^{2}}} .
$$


Thus $g_{q^{2}}$ can be modified by left multiplication by any element of the form $g \gamma(g)$. The sequence will split when one of these candidates for $g_{q^{2}}$ is the identity.

To summarize:

Lemma 2.2. Let $\gamma \in$ Aut $D_{n}$. If $\gamma$ is admissible, there are extensions

$$
1 \rightarrow D_{n} \rightarrow G \rightarrow \mathbb{Z}_{2} \rightarrow 1
$$

in which the conjugation action of some $q \in G \backslash D_{n}$ is given by $\gamma$. If $n=2$, the extensions are

$$
1 \rightarrow D_{2} \rightarrow D_{4} \rightarrow \mathbb{Z}_{2} \rightarrow 1
$$

with $D_{2}$ mapping to $\left\langle s^{2}, t\right\rangle \subset D_{4}$, and the two abelian extensions with $G \approx \mathbb{Z}_{4} \times \mathbb{Z}_{2}$ and $G \approx\left(\mathbb{Z}_{2}\right)^{3}$. For $n>2$, there is exactly one such extension if $n$ is odd, and two if $n$ is even, and every extension is of this form. The resulting groups $G$ have presentations of the form

$$
\left.\left\langle D_{n}, q\right| q^{2}=g_{q^{2}}, q^{-1} g q=\gamma(g) \text { for } g \in D_{n}\right\rangle .
$$

Let Tet, Oct, and Icos denote the three symmetry groups of the Platonic solids.

Lemma 2.3. Let $K$ be one of the groups Tet, Oct, or Icos. Then every extension

$$
1 \rightarrow K \rightarrow G \rightarrow \mathbb{Z}_{2} \rightarrow 0
$$

is split.

Proof. Recall that Tet $\approx A_{4}$, Oct $\approx S_{4}$, and Icos $\approx A_{5}$. A group $G$ is said to be complete if $Z(G)$ is trivial and every automorphism of $G$ is inner. For $n \neq 2,6$, $S_{n}$ is complete; see [Rotman 1995, Theorem 7.5]. Alternating groups are never complete: for $n>2, A_{n} \subseteq S_{n}$, and conjugation by the transposition (12) is an automorphism of $A_{n}$ which is not inner in $A_{n}$.

Claim. Aut $A_{4} \approx S_{4}$.

We know $S_{4} \subseteq$ Aut $A_{4}$, since any conjugation by an element of $S_{4}$ leaves $A_{4}$ invariant, and since $Z\left(S_{4}\right)$ is trivial. $A_{4}$ has the presentation $\langle x, y| x^{3}=y^{2}=$ $\left.(x y)^{3}=1\right\rangle$. Any automorphism must send $x$ to an element of order 3 , and $y$ to an element of order 2. Since $A_{4}$ contains 8 elements of order 3 and 3 elements of order 2, we see that $\mid$ Aut $A_{4} \mid \leq 3 \cdot 8=24$. But $\left|S_{4}\right|=24$.

Claim. Aut $A_{5} \approx S_{5}$.

This follows by an argument similar to the preceding one: the presentation $\left\langle x, y \mid x^{2}=y^{5}=(x y)^{3}=1\right\rangle$ gives an upper bound $\mid$ Aut $A_{5} \mid \leq 180$. But Aut $A_{5} \supseteq S_{5}$, a group of order 120 .

Thus Out Tet $\approx$ Out Icos $\approx \mathbb{Z}_{2}$, with the outer automorphism realized by conjugation by a transposition in $S_{4}$ or $S_{5}$. Out Oct is trivial, since $S_{4}$ is complete. 
Now, each of Tet, Oct, and Icos has trivial center, so in each case,

$$
H^{2}\left(\mathbb{Z}_{2} ; Z(K)\right)=0 .
$$

Thus if an abstract kernel admits a realization, it will be unique. Extensions do exist in all cases: the two extensions of Tet have $G \cong S_{4}$ and $G \cong$ Tet $\times \mathbb{Z}_{2}$; the extensions of Icos have $G \cong S_{5}$ and $G \cong$ Icos $\times \mathbb{Z}_{2}$; and the unique extension of Oct has $G \cong$ Oct $\times \mathbb{Z}_{2}$. All are split extensions.

The last classification lemma is somewhat more technical than the others, but it turns out to be exactly what is necessary when $\varphi(G)=\mathbb{Z}_{2} \times \mathbb{Z}_{2}$.

Lemma 2.4. Suppose $\langle a, b\rangle \cong \mathbb{Z}_{2} \times \mathbb{Z}_{2}$, and $1 \rightarrow \mathbb{Z}_{n} \rightarrow G \stackrel{\varphi}{\rightarrow}\langle a, b\rangle \rightarrow 1$ is exact. Let $G_{a}=\varphi^{-1}(\langle a\rangle)$ and $G_{b}=\varphi^{-1}(\langle b\rangle)$. Finally, let $\gamma=\mu_{q_{b}}$, viewed as an automorphism of $G_{a}$. Using the notation of Lemma 2.1, we have $\left.\gamma\right|_{\mathbb{Z}_{n}}=$ $\left(\delta_{2}, \epsilon_{2}, \ldots, \epsilon_{p_{i}}, \ldots, \epsilon_{p_{k}}\right)$. If $G_{a}$ is abelian and $1 \rightarrow G_{a} \rightarrow G \rightarrow\langle b\rangle \rightarrow 1$ does not split, then one of the following holds:

(1) $G \cong D_{2^{n_{2} m_{-}}}^{*} \times \mathbb{Z}_{m_{+}}$, with $\epsilon_{2}=-1, G_{a} \cong \mathbb{Z}_{2 n}$, and $\gamma\left(q_{a}\right)=q_{a}^{-1}$.

(2) $G \cong\left(\left(\mathbb{Z}_{m_{-}} \rtimes \mathbb{Z}_{2^{n_{2}+1}}\right) \times \mathbb{Z}_{m_{+}}\right) \rtimes \mathbb{Z}_{2}$, with $G_{a} \cong \mathbb{Z}_{a} \times \mathbb{Z}_{2}, n_{2}>1, \epsilon_{2}=1$, and $\gamma\left(q_{a}\right)=((n / 2) k) q_{a}$.

(3) $G \cong\left(D_{2^{n_{2}-1} m_{-}}^{*} \times \mathbb{Z}_{m_{+}}\right) \times \mathbb{Z}_{2}$, with $G_{a} \cong \mathbb{Z}_{n} \times \mathbb{Z}_{2}, \epsilon_{2}=-1$, and $\gamma\left(q_{a}\right)=q_{a}$.

(4) $G \cong\left(\mathbb{Z}_{m_{-}} \rtimes \mathbb{Z}_{2^{n_{2}+1}}\right) \times \mathbb{Z}_{m_{+}} \times \mathbb{Z}_{2}$, with $G_{a} \cong \mathbb{Z}_{n} \times \mathbb{Z}_{2}, \epsilon_{2}=1$, and $\gamma\left(q_{a}\right)=q_{a}$.

In the latter two cases, $q_{a}$ and $q_{b}$ commute. Notation, including some important normalizations for $q_{a}$ and $q_{b}$, is explained in the proof.

Proof. We use additive notation for the group operation in $K$, but multiplicative notation in the (possibly nonabelian) group $G$. Let $k$ generate $K=\mathbb{Z}_{n}$. $G$ is generated by $k$, together with $q_{a}$ and $q_{b}$, where $\varphi\left(q_{a}\right)=a$ and $\varphi\left(q_{b}\right)=b$. We assume $q_{a}$ and $q_{b}$ are normalized so that $g_{q_{a}^{2}}$ and $g_{q_{b}^{2}}$ lie in the Sylow 2-subgroup of $K$, and

(1) $g_{q_{a}^{2}}$ is either 0 , or a generator of this subgroup.

(2) If $\epsilon_{2}=1$, then $g_{q_{b}^{2}}$ is either 0 , or a generator.

(3) If $\epsilon_{2}=-1$, then either $g_{q_{b}^{2}}=0$, or it has order two.

With this in mind, the problem easily reduces to the case $n=2^{n_{2}}$, since the restriction of $\gamma$ to the subgroup $\mathbb{Z}_{m_{-} m_{+}}$of $\mathbb{Z}_{n}$ is known. We assume henceforth that $n$ is a power of 2 .

If $g_{q_{b}^{2}}=0$, the sequence splits, so we assume $g_{q_{b}^{2}} \neq 0$. Once $G_{a}$ is known, $G$ is determined by the automorphism $\gamma$ of $G_{a}$. In the proof of Lemma 2.1, we observed that $\left.\gamma\right|_{\mathbb{Z}_{n}}$ is described by a pair $\left(\delta_{2}, \epsilon_{2}\right) \in\{0,1\} \times\{ \pm 1\}$, where $k \mapsto\left(\epsilon_{2}+\delta_{2} \cdot 2^{n_{2}-1}\right) k$, and also that if $\delta_{2}=1$, then $1 \rightarrow \mathbb{Z}_{n} \rightarrow G_{b} \rightarrow \mathbb{Z}_{2} \rightarrow 1$ splits. So we may assume 
$\gamma(k)=\epsilon_{2} \cdot k$ and consider the various cases for $\gamma\left(q_{a}\right)$. Note that $\gamma\left(q_{a}\right)=c q_{a}$ for some $c \in K$, and that $\gamma\left(q_{a}^{2}\right)=2 c+g_{q_{a}^{2}}$. Hence $c$ is determined $\bmod 2^{n_{2}-1}$ by $\left.\gamma\right|_{\mathbb{Z}_{n}}$.

If $G_{a} \cong \mathbb{Z}_{2 n}$, then the argument cited in Lemma 2.1 shows that $1 \rightarrow G_{a} \rightarrow$ $G \rightarrow\langle b\rangle \rightarrow 1$ splits if $c \neq 0$. So we assume $\gamma\left(q_{a}\right)=q_{a}^{ \pm 1}$, depending on $\epsilon_{2}$. If $\epsilon_{2}=-1$, then $G \cong D_{2^{n_{2}}}^{*}$. If $\epsilon_{2}=1$, then $G \cong \mathbb{Z}_{2 n} \times \mathbb{Z}_{2}$, and $\left(q_{a} q_{b}^{-1}\right)^{2}=1$, so $1 \rightarrow G_{a} \rightarrow G \rightarrow\langle b\rangle \rightarrow 1$ splits.

If $G_{a} \cong \mathbb{Z}_{n} \times \mathbb{Z}_{2}$, we have several cases:

(1) If $c=0$ and $\epsilon_{2}=1$, then $G \cong \mathbb{Z}_{2^{n_{2}+1}} \times \mathbb{Z}_{2}$. $G$ contains three elements of order 2 , but all are contained in $G_{a}$, so the sequence $1 \rightarrow G_{a} \rightarrow G \rightarrow\langle b\rangle \rightarrow 1$ does not split.

(2) If $c=0$ and $\epsilon_{2}=-1$, then $G \cong G_{b} \times \mathbb{Z}_{2} \cong D_{2^{n_{2}-1}}^{*} \times \mathbb{Z}_{2}$.

(3) If $c=2^{n_{2}-1} k$ and $\epsilon_{2}=1$, then $G_{b} \cong \mathbb{Z}_{2^{n_{2}+1}}$, and $G \cong G_{b} \rtimes \mathbb{Z}_{2}=\mathbb{Z}_{2^{n_{2}+1}} \rtimes \mathbb{Z}_{2}$, with $q_{a}^{-1} q_{b} q_{a}=\left(\left(1+2^{n_{2}-1}\right) k\right) q$. Although $G$ contains involutions, the extension $1 \rightarrow G_{a} \rightarrow G \rightarrow\langle b\rangle \rightarrow 1$ does not split if $n_{2}>1$.

(4) If $c=2^{n_{2}-1} k$ and $\epsilon_{2}=-1$, then $\left(q_{a} q_{b}\right)^{2}=1$, so the sequence splits.

In Section 4, we will require explicit descriptions of the restriction maps $r^{*}$ : $H^{2}\left(D_{4} ; \mathbb{Z}\right) \rightarrow H^{2}(H ; \mathbb{Z})$ as $H$ ranges over the various subgroups of $D_{4}$. Rather than interrupt the flow of that argument later, we discuss them here. The methods used to calculate these maps are described in [Pearson 1996]. Some of the specific maps are also described there, and most of the rest were worked in the course of a conversation with the author of that paper.

Using the presentation $D_{4} \cong\left\langle s, t \mid s^{4}=t^{2}=1, t s t=s^{-1}\right\rangle$, the subgroups (up to conjugacy) can be enumerated as follows, with subscripts denoting generators: $G_{s} \approx \mathbb{Z}_{4}, G_{s^{2}} \approx \mathbb{Z}_{2}, G_{t} \approx \mathbb{Z}_{2}, G_{s t} \approx \mathbb{Z}_{2}, G_{s^{2}, t} \approx \mathbb{Z}_{2} \times \mathbb{Z}_{2}$, and $G_{s^{2}, s t} \approx \mathbb{Z}_{2} \times \mathbb{Z}_{2}$. The integral cohomology of these groups is computed from (known) descriptions of the cohomology with $\mathbb{Z}_{2}$ coefficients using the Bockstein spectral sequence.

For a general group $K, H^{1}\left(K ; \mathbb{Z}_{2}\right) \cong \operatorname{hom}\left(K_{a b}^{\prime} \mathbb{Z}_{2}\right)$, and generators of $H^{2}\left(K ; \mathbb{Z}_{2}\right)$ are often products of 1-dimensional classes. When integral cohomology classes are lifts of powers of $\mathbb{Z}_{2}$-classes, we will name them accordingly, even when the integral classes themselves are indecomposable.

Generators of $H^{1}\left(D_{4} ; \mathbb{Z}_{2}\right) \approx \operatorname{hom}\left(H_{1}\left(D_{4}\right), \mathbb{Z}_{2}\right)$ are given by $e$ and $f$, where $e(\bar{s})=1, e(\bar{t})=0, f(\bar{s})=1$, and $f(\bar{t})=1$. This seemingly asymmetrical choice of generators yields the convenient relation $e \cup f=0$, while $e^{2}$ and $f^{2}$ lift to generators $\widehat{e^{2}}$ and $\widehat{f^{2}}$ of $H^{2}\left(D_{4} ; \mathbb{Z}\right) \approx \mathbb{Z}_{2} \times \mathbb{Z}_{2} .\left(H_{2}\left(D_{4} ; \mathbb{Z}_{2}\right)\right.$ also contains an indecomposable element $w$ which does not lift.)

Similarly, for each copy of $\mathbb{Z}_{2} \times \mathbb{Z}_{2}$ described by an ordered set of generators $\langle x, y\rangle$, we have $H^{2}\left(\mathbb{Z}_{2} \times \mathbb{Z}_{2} ; \mathbb{Z}\right) \approx \mathbb{Z}_{2} \times \mathbb{Z}_{2}=\left\langle\widehat{a^{2}}, \widehat{b^{2}}\right\rangle$, where $a(x)=1, a(y)=0$, 
$b(x)=0$, and $b(y)=1$. A word of warning: we use the same notation for $H^{2}$ generators of different copies of $\mathbb{Z}_{2} \times \mathbb{Z}_{2}$; meaning can be determined from context.

Finally, $H^{2}\left(\mathbb{Z}_{4} ; \mathbb{Z}\right) \approx \mathbb{Z}_{4}$, with a generator denoted by $c$. Both $c$ and its reduction mod 2 are indecomposable.

Lemma 2.5. The restriction maps $r^{*}: H^{2}\left(D_{4} ; \mathbb{Z}\right) \rightarrow H^{2}(H ; \mathbb{Z})$, as $H$ ranges over subgroups of $D_{4}$, are as follows:

\begin{tabular}{c|cccccc} 
& $G_{s^{2}, t}$ & $G_{s^{2}, s t}$ & $G_{s^{2}}$ & $G_{t}$ & $G_{s t}$ & $G_{s}$ \\
\hline$\widehat{e^{2}}$ & 0 & $\widehat{b^{2}}$ & 0 & 0 & $\widehat{b^{2}}$ & $2 c$ \\
$\widehat{f^{2}}$ & $\widehat{b^{2}}$ & 0 & 0 & $\widehat{b^{2}}$ & 0 & $2 c$
\end{tabular}

Proof. We use the commutative diagram

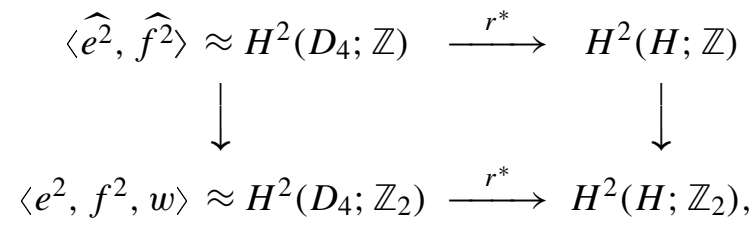

where the vertical maps are induced by the coefficient reduction. The right-hand map is an isomorphism for all the subgroups except for $\mathbb{Z}_{4}$, where $H^{2}\left(\mathbb{Z}_{4} ; \mathbb{Z}_{2}\right) \cong$ $H^{2}\left(\mathbb{Z}_{4} ; \mathbb{Z}\right) \otimes \mathbb{Z}_{2}$. The fact that $e^{2}$ and $f^{2}$ are squares of one-dimensional classes makes calculation easy for every column except $G_{s}$. In that case, since the generator of $H_{2}\left(\mathbb{Z}_{4} ; \mathbb{Z}_{2}\right)$ is indecomposable, the map $r^{*}: H_{2}\left(D_{4} ; \mathbb{Z}_{2}\right) \rightarrow H_{2}\left(\mathbb{Z}_{4} ; \mathbb{Z}_{2}\right)$ is zero. It follows immediately that each of $r^{*}\left(\widehat{e^{2}}\right)$ and $r^{*}\left(\widehat{f^{2}}\right)$ is either 0 or $2 c$. Determining which actually occurs requires a closer look at the Bockstein spectral sequence. Details are in [Pearson 1996]; our calculation later on actually only requires that each be an even multiple of $c$.

\section{The linear case}

Let us say that the group of linear actions on $S^{2} \times S^{2}$ is $W=\{A \in \mathrm{SO}(6) \mid$ $\left.A\left(S^{2} \times S^{2}\right)=S^{2} \times S^{2}\right\}$. What is the structure of $W$ ? The homology representation $\varphi$ extends to all of $W$. We will apply it in the proof of the following:

Lemma 3.1. $W \cong\left(\mathrm{SO}(3) \times \mathrm{SO}(3) \times \mathbb{Z}_{2}\right) \rtimes \mathbb{Z}_{2}$, where the semidirect product automorphism is a coordinate switch in the factors of $\mathrm{SO}(3) \times \mathrm{SO}(3)$.

Proof. The main claim that needs to be established is the exactness of the sequence

$$
1 \rightarrow \mathrm{SO}(3) \times \mathrm{SO}(3) \stackrel{i}{\rightarrow} W \stackrel{\varphi}{\rightarrow} \mathbb{Z}_{2} \times \mathbb{Z}_{2} \rightarrow 1 .
$$

Since a rotation in $\mathrm{SO}(3)$ is homotopic to the identity, any $g \in \mathrm{SO}(3) \times \mathrm{SO}(3)$ is in the kernel of $\varphi$. For the reverse inclusion, suppose $\varphi(g)=e \in \mathbb{Z}_{2} \times \mathbb{Z}_{2}$. Since $g$ 
acts trivially on homology, it has a fixed point $(x, y) \in S^{2} \times S^{2}$. A fairly standard calculation shows that the only sections of $T_{(x, y)} S^{2} \times S^{2}$ with sectional curvature $K=1$ are those tangent to the two factors. Since $g$ is an isometry, it preserves the splitting of $T_{(x, y)} S^{2} \times S^{2}$, so it acts on $T_{(x, y)} S^{2} \times S^{2}$ by a pair of rotations $\theta_{1}$ and $\theta_{2}$. Thus $g$ agrees with an element of $\mathrm{SO}(3) \times \mathrm{SO}(3)$ on a 5 -dimensional subspace of $\mathbb{R}^{6}$. Since $\operatorname{det}(g)=1, g \in \mathrm{SO}(3) \times \mathrm{SO}(3)$. This finishes the proof of exactness.

Now, the quotient $\mathbb{Z}_{2} \times \mathbb{Z}_{2}$ is easily seen to be generated by $\alpha$, the isometry which acts by the antipodal map in each factor, and $\sigma$, which switches coordinates. $\alpha$ is central in $\mathrm{SO}(6)$, so $\langle\alpha\rangle$ extends $\mathrm{SO}(3) \times \mathrm{SO}(3)$ with a direct product. However, $\sigma$ is not central, so the product is only semidirect.

For the remainder of this section, let $G$ be a finite subgroup of $W$ which acts linearly and pseudofreely and preserves orientation. It is well known (see [Wolf 1984; Kulkarni 1982], for example, or Lemma 4.4 below) that the finite groups which act pseudofreely on $S^{2}$ are either cyclic, dihedral, or one of the three symmetry groups of the Platonic solids. These groups also act pseudofreely on $S^{2} \times S^{2}$ via the diagonal action. It will turn out that if the induced action on homology is trivial, this is essentially, but not exactly, the only possibility. If the homology action is nontrivial, things are more complicated, and we approach them via the short exact sequence $1 \rightarrow K \rightarrow G \stackrel{\varphi}{\rightarrow} Q \rightarrow 1$.

Example. Consider the action of $\mathbb{Z}_{5}$ on $S^{2} \times S^{2}$ defined by $\gamma \cdot(x, y)=\left(\gamma \cdot x, \gamma^{2} \cdot y\right)$, where $\gamma$ acts on $S^{2}$ by a rotation of $2 \pi / 5$ around an axis. The action has four isolated fixed points. Notice also that this action resembles the diagonal action, in the sense that $\gamma \cdot(x, y)=(\gamma \cdot x, \psi(\gamma) \cdot y)$, where $\psi$ is the automorphism of $\mathbb{Z}_{5}$ sending $\gamma \mapsto \gamma^{2}$. However, this action is not equivalent to the diagonal action: A neighborhood of a singular point in the quotient by the diagonal action is a cone on the lens space $L(5,1)$, while the corresponding neighborhood for this action is a cone on $L(5,2)$.

Definition. Suppose $G$ acts on a space $X$. An action $\theta$ of $G$ on $X \times X$ will be called semidiagonal if there is an automorphism $\psi$ of $G$ so that $\theta$ is equivalent to $(g,(x, y)) \mapsto(g x, \psi(g)(y))$.

Proposition 3.2. If $G$ acts trivially on homology, then $G$ is a polyhedral group, and its action is semidiagonal.

Proof. Since $G$ acts trivially on homology, we must have $G \subset \mathrm{SO}(3) \times \mathrm{SO}(3)$. Let $(g, h) \in G$. Since each of $g$ and $h$ preserves orientation on $S^{2}$, each has a fixed point on $S^{2}$. By the assumption of pseudofreeness, each fixed set must be 0 -dimensional unless $(g, h)=(e, e)$.

Claim. Let $\pi_{1}$ and $\pi_{2}$ denote the two obvious projections from $\mathrm{SO}(3) \times \mathrm{SO}(3) \rightarrow$ $\mathrm{SO}(3)$. Then $\pi_{1}(G) \approx \pi_{2}(G)$. 
Observe that for any $g$, there is a unique $h$ so that $(g, h) \in G$. For if $\left(g, h_{1}\right)$ and $\left(g, h_{2}\right)$ are both in $G$ and $h_{1} \neq h_{2}$, then $\left(g^{-1}, h_{1}^{-1}\right)\left(g, h_{2}\right)=\left(1, h_{1}^{-1} h_{2}\right) \in G$. But $h_{1}^{-1} h_{2} \neq 1$, so it has two fixed points on $S^{2}$. But then Fix $\left(\left(1, h_{1}^{-1} h_{2}\right), S^{2} \times S^{2}\right) \cong$ $S^{2} \times S^{0}$, contradicting pseudofreeness.

Now we can define $\psi: \pi_{1}(G) \rightarrow \pi_{2}(G)$ by

$$
g \mapsto \text { the unique } h \text { such that }(g, h) \in G \text {. }
$$

$\psi$ is clearly a homomorphism, and by symmetry, an isomorphism. This proves the claim. To simplify notation, write $G_{1}=\pi_{1}(G)$ and $G_{2}=\pi_{2}(G)$.

$G_{1}$ acts pseudofreely on $S^{2}$, so it must be a polyhedral group. Moreover, the projection $G \rightarrow G_{1}$ must be injective, since if $(g, h) \in \operatorname{ker} \pi_{1}$, then $g=e$, and $h=\psi(g)=e$, also. Similar considerations apply to $G_{2}$. Now, any two isomorphic finite subgroups of $\mathrm{SO}(3)$ are conjugate [Wolf 1984, Theorem 2.6.5]. That is, there is a change of coordinates with respect to which we actually have $G_{1}=G_{2}$. Without loss of generality, assume we have applied it. (In doing so, we have fixed, once and for all, a particular identification between the two factors of $S^{2} \times S^{2}$. For later reference, this also defines a particular choice of coordinate switch $\sigma$ : $(x, y) \mapsto(y, x)$. Abstractly, $\sigma$ is only well-defined $\bmod \{e\} \times \mathrm{SO}(3)$.) Then for any $g \in G$, we have $g \cdot(x, y)=(g x, \psi(g) y)$.

Here it is worthwhile to note a consequence of the proof of Lemma 2.3: If the automorphism $\psi$ of $G$ above is inner, then after applying the coordinate change, we may treat $\psi$ as identity. Since Out Oct is trivial, every Oct action is diagonal. And since Out Tet $\approx$ Out Icos $\approx \mathbb{Z}_{2}$, each of Tet and Icos admits at most one nondiagonal action. In fact, by embedding Tet in Oct, we see that the outer automorphism of Tet is realized by an $\mathrm{SO}(3)$ conjugation, so every Tet action is diagonal. However, if there were an $\mathrm{SO}(3)$ conjugation which realized the outer automorphism of Icos, then $S_{5}$ would be a subgroup of $\mathrm{SO}(3)$. It isn't, so there are two linear, pseudofree actions of Icos which are not linearly equivalent.

If the action of $G$ on homology is nontrivial, what can we say? If $G \subset O(3) \times$ $O(3)$, for example, the only possible nontrivial action is via $\left(\begin{array}{rr}-1 & 0 \\ 0 & -1\end{array}\right)$. Thus we have a short exact sequence

$$
1 \rightarrow K \rightarrow G \rightarrow \mathbb{Z}_{2} \rightarrow 0
$$

Since we know the possible groups $K$ in the above sequence, we should expect the possible groups $G$ simply to be extensions of polyhedral groups by $\mathbb{Z}_{2}$. But it turns out that many of the extended actions can not be pseudofree and must be ruled out. The following statement describes the possible groups $G$. Explicit actions are constructed in its proof. 
Proposition 3.3. If the homology action of $G$ is given by $\varphi(G) \approx\left\langle\left(\begin{array}{rr}-1 & 0 \\ 0 & -1\end{array}\right)\right\rangle$, then $G \approx \mathbb{Z}_{n} \times \mathbb{Z}_{2}$ or $G \approx \mathbb{Z}_{2 n}$. Pseudofree actions exist in these cases.

Proof. We begin with some observations about the geometry of $W$.

Suppose $g$ and $s$ are nontrivial elements of $\mathrm{SO}(3)$. Each has a well defined axis of rotation - we denote them respectively by $X_{g}$ and $X_{s}$. Observe that $\mu_{g}(s)=s^{l}$ for some $l$ if and only if $g$ leaves $X_{s}$ invariant. If $g$ fixes $X_{s}$, then $X_{g}=X_{s}$, and $\mu_{g}(s)=s$. If $g$ inverts $X_{s}$, then $X_{s} \perp X_{g}$, and $g$ is an order 2 rotation. In this case, $\mu_{g}(s)=s^{-1}$.

Lemma 3.1 tells us that any $q \in W$ with $\varphi(q)=\left(\begin{array}{rr}-1 & 0 \\ 0 & -1\end{array}\right)$ has the form $q=$ $(\alpha, \alpha)\left(r_{1}, r_{2}\right)$, where $\left(r_{1}, r_{2}\right) \in \mathrm{SO}(3) \times \mathrm{SO}(3)$. Also, an element $S \in W$ acting homologically trivially and pseudofreely has the form $\left(s_{1}, s_{2}\right)$, where neither coordinate is 1 . It follows from the previous observations that if $q^{2}=1$ and $\mu_{q}(s) \neq s$ for some $s$, then $r_{1}$ and $r_{2}$ are both nontrivial order 2 rotations, and $q$ fixes a torus. So if $G$ acts pseudofreely, any $q$ with $\varphi(q)=\left(\begin{array}{rr}-1 & 0 \\ 0 & -1\end{array}\right)$ and $q^{2}=1$ must be central in $G$. Similar considerations show that a $q$ of order greater than 2 leaves invariant a unique axis in each factor, so at most one cyclic subgroup of $K$ can be normalized by $q$, and in fact, the generator of such a group commutes with $q$. Thus for each $q \in G \backslash K$, one of the following holds:

(1) $q$ has order 2 , and $\mu_{q}$ is trivial.

(2) $q$ has order greater than 2 , and $q$ normalizes (at most) a single maximal cyclic subgroup of $K$, which it centralizes.

Now consider the possibilities for $K$ :

Case 1: $K \cong \mathbb{Z}_{n}$. The only groups $G$ satisfying conditions (1) and (2) are $G \cong$ $\mathbb{Z}_{n} \times \mathbb{Z}_{2}$ and $G \cong \mathbb{Z}_{2 n}$. If $n$ is odd, these two groups are isomorphic, and the extension is realized by choosing $q=(\alpha, \alpha) \circ(1, r)$, where $r$ has order 2 and shares an axis with the generator of $K$. If $n$ is even, this construction realizes the case $\mathbb{Z}_{n} \times \mathbb{Z}_{2} . \mathbb{Z}_{2 n}$ is realized if both $r_{1}$ and $r_{2}$ are order $2 n$ rotations. Note that if $n$ is odd, the latter construction still yields a $\mathbb{Z}_{2 n}$ action, but if $n$ is odd, then $q^{n}$ fixes a torus.

Case 2: $K \cong D_{n}=\left\langle s, t \mid s^{n}=t^{2}=1, t s t=s^{-1}\right\rangle$. If some $q$ exists satisfying condition (2), then $t q$ has order 2 but fails to satisfy (1). If no $q$ satisfies (2), then $n=2$, and either $q, s q, t q$, or $s t q$ has both $r_{1}$ and $r_{2}$ nontrivial, and thus fixes a torus.

Case 3: $K \cong T e t$, Oct, or Icos. By Lemma 2.3, the sequence $1 \rightarrow K \rightarrow G \rightarrow \mathbb{Z}_{2} \rightarrow 1$ splits, so we may pick $q$ of order 2 . By condition (1), $\mu_{q}$ is trivial. Now let $g \in K$ be an involution. Then $g q$ also has order 2 , but $\mu_{g q}$ is nontrivial, contradicting condition (1). 
Next we consider the case in which $\varphi(G) \approx\left\langle\left(\begin{array}{ll}0 & 1 \\ 1 & 0\end{array}\right)\right\rangle$. For actions which need not be locally linear, it follows from a theorem of Bredon that:

Proposition 3.4. (1) If the sequence $1 \rightarrow K \rightarrow G \rightarrow\left\langle\left(\begin{array}{ll}0 & 1 \\ 1 & 0\end{array}\right)\right\rangle \rightarrow 1$ splits, then $G$ can not act pseudofreely.

(2) Suppose $G$ acts on $S^{2} \times S^{2}$ with $\varphi(G) \cong \mathbb{Z}_{2}$. If the action of $K=\operatorname{ker} \varphi$ is pseudofree, and $1 \rightarrow K \rightarrow G \rightarrow \mathbb{Z}_{2} \rightarrow 1$ does not split, then the action of $G$ is pseudofree.

Proof. As a special case of Bredon's theorem [1972, VII.7.5], an involution $T$ on $S^{2} \times S^{2}$ with $T^{*} \neq 1$ on $H^{2}\left(S^{2} \times S^{2} ; \mathbb{Z}_{2}\right)$ has a fixed point set $F$ with $H^{2}\left(F ; \mathbb{Z}_{2}\right)=$ $\mathbb{Z}_{2}$. If the sequence $1 \rightarrow K \rightarrow G \rightarrow\left\langle\left(\begin{array}{ll}0 & 1 \\ 1 & 0\end{array}\right)\right\rangle \rightarrow 1$ splits, then $G$ contains such an involution. The second statement follows from the observation that for $q \in G \backslash K$, $\operatorname{Fix}\left(q^{2}\right) \subseteq \operatorname{Fix}(q)$.

Corollary 3.5. If the homology action of $G$ is given by $\varphi(G) \approx\left\langle\left(\begin{array}{ll}0 & 1 \\ 1 & 0\end{array}\right)\right\rangle$, then $K$ can't be Tet, Oct, or Icos.

Proof. This follows immediately from Lemma 2.3.

Proposition 3.6. If $K \cong \mathbb{Z}_{n}$ and $\varphi(G) \approx\left\langle\left(\begin{array}{ll}0 & 1 \\ 1 & 0\end{array}\right)\right\rangle$, then either

(1) $G \cong\left(\mathbb{Z}_{m_{-}} \rtimes \mathbb{Z}_{2^{n_{2}+1}}\right) \times \mathbb{Z}_{m_{+}}$or

(2) $G \cong D_{2^{n^{2}-1} m_{-}}^{*} \times \mathbb{Z}_{m_{+}}$,

with notation as in the proof of Lemma 2.1. Pseudofree linear actions exist in these cases.

Proof. We know from Proposition 3.4 that if $1 \rightarrow K \rightarrow G \rightarrow \mathbb{Z}_{2} \rightarrow 1$ splits, then $G$ cannot act pseudofreely. It follows from Lemma 2.1 that $G$ must be of type (1) or (2) (and that $n$ is even). Recall from the beginning of Section 2 that the structure of $G$ is determined by the data $\gamma$ and $g_{q^{2}}$. By Lemma 3.1, a hypothetical $q \in G \backslash K$ has the form $\sigma r$, where $\sigma:(x, y) \mapsto(y, x)$, and $r=\left(r_{1}, r_{2}\right) \in \mathrm{SO}(3) \times \mathrm{SO}(3)$. Our construction of a pseudofree $G$-action uses two ingredients:

(1) A choice of a particular semidiagonal $K$-action, which makes it possible to realize $\gamma$ as $\mu_{q}$.

(2) An appropriate choice of $r$ which ensures that $q^{2}=g_{q^{2}}$.

As in the proof of Proposition 3.3, conjugation by $r$ must either fix or invert the axis of rotation of $K$ in each factor, so $\mu_{\sigma r}=\mu_{r} \circ \mu_{\sigma}$ is either (inversion) $\circ \mu_{\sigma}$, or simply $\mu_{\sigma}$. For simplicity, we will assume that $r_{1}$ and $r_{2}$ share the axis of $K$, so $\mu_{\sigma r}=\mu_{\sigma}$. The construction can also be carried out with minor modifications if $X_{r_{i}} \perp X_{K}$.

A semidiagonal embedding of $K \subset \mathrm{SO}(3)$ into $\mathrm{SO}(3) \times \mathrm{SO}(3)$ takes the form $k \stackrel{i}{\mapsto}(k, \psi(k)) \in \mathrm{SO}(3) \times \mathrm{SO}(3) \quad$ for some $\psi \in$ Aut $K$. 
Observe that $\mu_{\sigma}(k, \psi(k))=\sigma(k, \psi(k)) \sigma=(\psi(k), k)$, and also that $i(\gamma(k))=$ $(\gamma(k), \psi(\gamma(k)))$. Since $\gamma^{2}=1$, choosing $\psi=\gamma$ allows us to realize $\gamma$ as $\mu_{q}$ :

$$
\mu_{q}(i(k))=\mu_{\sigma}(k, \psi(k))=(\gamma(k), k)=(\gamma(k), \psi(\gamma(k)))=i(\gamma(k)) .
$$

Next observe that $q^{2}=\sigma r \sigma r=\left(r_{2} r_{1}, r_{1} r_{2}\right)=\left(r_{1} r_{2}, r_{1} r_{2}\right)$. Thus if we choose $r_{1}$ and $r_{2}$ so that $r_{1} r_{2}=g_{q^{2}}$, then $q^{2}=\left(g_{q^{2}}, g_{q^{2}}\right)=\left(g_{q^{2}}, \psi\left(g_{q^{2}}\right)\right)=i\left(g_{q^{2}}\right)$ (recall that $\psi=\gamma$ fixes $g_{q^{2}}$ ).

It follows from the second part of Proposition 3.4 that the extended action is still pseudofree.

Proposition 3.7. If $K \cong D_{n}$ and $\varphi(G) \cong\left\langle\left(\begin{array}{ll}0 & 1 \\ 1 & 0\end{array}\right)\right\rangle$, then $G$ acts pseudofreely and linearly if and only if the sequence $1 \rightarrow K \rightarrow G \rightarrow \mathbb{Z}_{2} \rightarrow 1$ does not split.

Proof. We know it is necessary that the sequence not split. To prove the converse, we assume it does not split and we construct a pseudofree $G$-action. Let the data $\gamma$ and $g_{q^{2}}$ be given. As in the previous proof, $q=\sigma r$, and we will choose $r=\left(r_{1}, r_{2}\right)$ and $\psi \in$ Aut $D_{n}$ appropriately. In this case, choose any $r_{1}$ and $r_{2}$ so that $r_{2} r_{1}=$ $g_{q^{2}} \in D_{n} \subset \mathrm{SO}(3)$, and each $\mu_{r_{i}}$ leaves $D_{n}$ invariant. (For example, simply take $r_{1}=1, r_{2}=g_{q^{2}}$.) Let $\psi=\mu_{r_{2}} \circ \gamma^{-1} \in$ Aut $D_{n}$, and let $i$ embed $D_{n}$ in $\mathrm{SO}(3) \times \operatorname{SO}(3)$ via $g \stackrel{i}{\mapsto}(g, \psi(g))$.

We show that the subgroup of $W$ generated by $i\left(D_{n}\right)$ and $q$ is isomorphic to $G$, and then appeal to Proposition 3.4 to see that the resulting action of $G$ is pseudofree.

For the first claim, it suffices to verify that $q^{2}=i\left(g_{q^{2}}\right)$ and that $\mu_{q} \circ i=$ $i \circ \gamma$. We have $q^{2}=\left(r_{2} r_{1}, r_{1} r_{2}\right)$. On the other hand, $i\left(g_{q^{2}}\right)=\left(r_{2} r_{1}, \psi\left(r_{2} r_{1}\right)\right)=$ $\left(r_{2} r_{1}, r_{2}^{-1}\left(\gamma^{-1}\left(r_{2} r_{1}\right)\right) r_{2}\right)=\left(r_{2} r_{1}, r_{1} r_{2}\right)$, because $\gamma$ fixes $g_{q^{2}}$. Note that as a consequence, $\mu_{r_{2} r_{1}}=\mu_{g_{q^{2}}}=\gamma^{2}$.

For $g \in D_{n}, i(\gamma(g))=(\gamma(g), \psi(\gamma(g)))=\left(\gamma(g), r_{2}^{-1} g r_{2}\right)$. But $\mu_{q}(i(g))=$ $\mu_{q}(g, \psi(g))=\mu_{r}\left(\mu_{\sigma}(g, \psi(g))\right)=\mu_{r}(\psi(g), g)=\left(r_{1}^{-1} r_{2}^{-1} \gamma^{-1}(g) r_{2} r_{1}, r_{2}^{-1} g r_{2}\right)=$ $\left(\gamma^{2}\left(\gamma^{-1}(g)\right), r_{2}^{-1} g r_{2}\right)=\left(\gamma(g), r_{2}^{-1} g r_{2}\right)$.

Proposition 3.8. Suppose $\varphi(G)=\mathbb{Z}_{2} \times \mathbb{Z}_{2}$. Then

(1) $G \cong D_{2^{n_{2} m_{-}}}^{*} \times \mathbb{Z}_{m_{+}}$, or

(2) $G \cong\left(\left(\mathbb{Z}_{m_{-}} \rtimes \mathbb{Z}_{2^{n_{2}+1}}\right) \times \mathbb{Z}_{m_{+}}\right) \rtimes \mathbb{Z}_{2}$, with $n_{2}>1$,

with notation as in Lemma 2.1. Pseudofree actions exist in these cases.

Proof. Let $a=\left(\begin{array}{rr}-1 & 0 \\ 0 & -1\end{array}\right)$ and $b=\left(\begin{array}{ll}0 & 1 \\ 1 & 0\end{array}\right) \in$ Aut $H_{2}\left(S^{2} \times S^{2}\right)$, and suppose an extension

$$
1 \rightarrow H \rightarrow G \stackrel{\varphi}{\rightarrow}\langle a\rangle \times\langle b\rangle \rightarrow 1
$$

exists, with $G$ acting pseudofreely. Let $G_{a}=\varphi^{-1}(\langle a\rangle)$, and $G_{b}=\varphi^{-1}(\langle b\rangle)$. 
By Propositions 3.3 and 3.4, we know that $K$ must be cyclic of even order, with $G_{a} \cong \mathbb{Z}_{2 n}$ or $\mathbb{Z}_{n} \times \mathbb{Z}_{2}$, and that the sequence $1 \rightarrow G_{a} \rightarrow G \rightarrow\langle b\rangle \rightarrow 1$ must not split. Lemma 2.4 describes those groups $G$ which satisfy these criteria.

If $G_{a} \cong \mathbb{Z}_{n} \times \mathbb{Z}_{2}$, the geometry of $W$ puts one more restriction on $G$. Let $q_{a}^{2}=1$, where $\varphi\left(q_{a}\right)=a$. Then $q_{a}=(\alpha, \alpha)\left(r_{1}, r_{2}\right)$, where $\left(r_{1}\right)^{2}=\left(r_{2}\right)^{2}=1$. If both $r_{1}$ and $r_{2}$ are nontrivial, then $q_{a}$ fixes a torus. If both are trivial, then the product of $q_{a}$ with the unique element of order two in $K$ fixes a torus. So exactly one of them is nontrivial. Suppose it is $r_{1}$. The element $q_{b}$ has the form $\sigma\left(s_{1}, s_{2}\right)$, so $q_{b}^{-1} q_{a} q_{b}=(\alpha, \alpha)\left(1, s_{2}^{-1} r_{1} s_{2}\right)$. Thus $q_{a}$ and $q_{b}$ can not commute in the linear case.

Lemma 2.4 then shows that $G$ necessarily has one of the forms given in the statement of the theorem. We must now construct pseudofree actions of these groups. For simplicity, we assume all rotations used in the constructions have the same axes.

Case 1: $G \cong D_{2^{n_{2} m_{-}}}^{*} \times \mathbb{Z}_{m_{+}}$. We start with an action of $G_{b}=D_{2^{n_{2}-1} m_{-}}^{*} \times \mathbb{Z}_{m_{+}}$as provided by Proposition 3.6. Extend $\psi$ (the semidiagonalizing automorphism of $K)$ to an automorphism of $\mathbb{Z}_{2 n}$. Let $q_{a}=(\alpha, \alpha)(r, \psi(r))$, where $r^{2}$ is a generator of the Sylow 2-subgroup of $K$. Then $\left\langle K, q_{a}\right\rangle \cong \mathbb{Z}_{2 n}$ acts pseudofreely. We need only verify that $q_{b}^{-1} q_{a} q_{b}=q_{a}^{-1}$ to conclude that the subgroup of $W$ generated by $G_{b}$ and $q_{a}$ is isomorphic to $G$ :

$$
\begin{aligned}
\left(s_{1}^{-1}, s_{2}^{-1}\right) \sigma(\alpha, \alpha)(r, \psi(r)) \sigma\left(s_{1}, s_{2}\right) & =(\alpha, \alpha)(\psi(r), r) \\
& =(\alpha, \alpha)\left(r^{-1}, \psi(r)^{-1}\right)=q_{a}^{-1} .
\end{aligned}
$$

Case 2: $G \cong\left(\left(\mathbb{Z}_{m_{-}} \rtimes \mathbb{Z}_{2^{n_{2}+1}}\right) \times \mathbb{Z}_{m_{+}}\right) \rtimes \mathbb{Z}_{2}$, with $n_{2}>1$. Again, start with an action of $G_{b}=\left(\mathbb{Z}_{m_{-}} \rtimes \mathbb{Z}_{2^{n_{2}+1}}\right) \rtimes \mathbb{Z}_{m_{+}}$as in Proposition 3.6. Let $q_{a}=(\alpha, \alpha)(r, 1)$, where $r$ is an order two rotation. Then $q_{a}^{2}=1$, so $G_{a}=\left\langle K, q_{a}\right\rangle \cong \mathbb{Z}_{n} \rtimes \mathbb{Z}_{2}$. And we have $q_{b}=\sigma\left(s_{1}, s_{2}\right)$, chosen so that $\left(q_{b}\right)^{2}=k$ generates the 2 -subgroup of $\mathbb{Z}_{n}$. To show that $\left\langle G_{b}, q_{a}\right\rangle \cong G$, we observe that

$$
q_{a}^{-1} q_{b} q_{a}=(\alpha, \alpha)(r, 1) \sigma\left(s_{1}, s_{2}\right)(\alpha, \alpha)(r, 1)=2^{n_{2}-1} k q_{a},
$$

as required.

Pseudofreeness of the extended actions follows from Proposition 3.4, as usual.

Gathering together the results of this section, we have:

Theorem 3.9. The following are all of the groups $G$ which act linearly and pseudofreely on $S^{2} \times S^{2}$ :

(1) Tet, Oct, Icos, $\mathbb{Z}_{n}$, and $D_{n}$, acting homologically trivially.

(2) $\mathbb{Z}_{n} \times \mathbb{Z}_{2}$ and $\mathbb{Z}_{2 n}$, where $K \approx \mathbb{Z}_{n}$, and $\varphi(G) \approx\left\langle\left(\begin{array}{rr}-1 & 0 \\ 0 & -1\end{array}\right)\right\rangle$.

(3) The groups 
(a) $G \cong\left(\mathbb{Z}_{m_{-}} \rtimes \mathbb{Z}_{2^{n_{2}+1}}\right) \times \mathbb{Z}_{m_{+}}$, or

(b) $G \cong D_{2^{n_{2}-1} m_{-}}^{*} \times \mathbb{Z}_{m_{+}}$,

(c) $\left\langle D_{n}, k\right| k^{2}=g_{k^{2}}, \mathrm{kgk}^{-1}=\gamma(\mathrm{g})$ for $\left.g \in D_{n}\right\rangle$, where $1 \rightarrow D_{n} \rightarrow G \rightarrow$ $\mathbb{Z}_{2} \rightarrow 1$ does not split.

In this case, $\varphi(G) \approx\left\langle\left(\begin{array}{ll}0 & 1 \\ 1 & 0\end{array}\right)\right\rangle$.

(4) The groups

(a) $G \cong D_{2^{n_{2} m_{-}}}^{*} \times \mathbb{Z}_{m_{+}}$, or

(b) $G \cong\left(\left(\mathbb{Z}_{m_{-}} \rtimes \mathbb{Z}_{2^{n_{2}+1}}\right) \times \mathbb{Z}_{m_{+}}\right) \rtimes \mathbb{Z}_{2}$, with $n_{2}>1$.

In this case, $\varphi(G) \approx \mathbb{Z}_{2} \times \mathbb{Z}_{2}$.

This theorem can be restated in a form somewhat more amenable to generalization to the nonlinear case: Let $a=\left(\begin{array}{cc}-1 & 0 \\ 0 & -1\end{array}\right)$ and $b=\left(\begin{array}{ll}0 & 1 \\ 1 & 0\end{array}\right) \in$ Aut $H_{2}\left(S^{2} \times S^{2}\right)$, and suppose an extension

$$
1 \rightarrow K \rightarrow G \stackrel{\varphi}{\rightarrow} Q \rightarrow 1
$$

exists, with $Q \subset\langle a\rangle \times\langle b\rangle$. The sequence then determines the subgroups $G_{a}=$ $\varphi^{-1}(\langle a\rangle)$, and $G_{b}=\varphi^{-1}(\langle b\rangle)$.

Conversely, suppose a homology representation of a group $G$ is described by a tuple $\left(G, K, G_{a}, G_{b}\right)$ as above.

Corollary 3.10. $\left(G, K, G_{a}, G_{b}\right)$ admits a linear, pseudofree action on $S^{2} \times S^{2}$ if and only if

(1) $K$ is polyhedral.

(2) If $G_{a} \neq K$, then $G_{a}$ is abelian and $K$ is cyclic.

(3) If $G_{b} \neq K$, then $1 \rightarrow G_{a} \rightarrow G \rightarrow \mathbb{Z}_{2} \rightarrow 1$ does not split.

(4) If $\varphi\left(q_{a}\right)=a, \varphi\left(q_{b}\right)=b$, and $q_{a}^{2}=1$, then $q_{a}$ and $q_{b}$ do not commute.

Proof. We have already seen that condition 1 is necessary and sufficient in the homologically trivial case, and that condition 2 is necessary and sufficient in the $\pm\left(\begin{array}{ll}1 & 0 \\ 0 & 1\end{array}\right)$ case. Condition 3 is necessary by Proposition 3.4 (Bredon's theorem). On the other hand, this condition implies that $1 \rightarrow H \rightarrow G_{b} \rightarrow \mathbb{Z}_{2} \rightarrow 1$ does not split, and Propositions 3.6 and 3.7 show this to be sufficient in the $\left(\begin{array}{ll}0 & 1 \\ 1 & 0\end{array}\right)$ case. Finally, Lemma 2.4 enumerates those groups $G$ with $\varphi(G) \cong \mathbb{Z}_{2} \times \mathbb{Z}_{2}$ which satisfy the first three conditions, and Proposition 3.8 shows that the last condition is necessary and sufficient to finish the classification.

\section{The nonlinear case}

In this section we prove that any group which acts pseudofreely and locally linearly also acts pseudofreely and linearly. (It should be pointed out that nonlinear actions definitely do exist: Edmonds and Ewing [1992] construct pseudofree actions of 
cyclic groups with fixed point data incompatible with linearity.) Our strategy follows the statement of Corollary 3.10: Bredon's theorem shows that condition 3 is necessary. We show that conditions 1,2, and 4 are necessary in the general case.

For the remainder of the paper, we will make constant use of the Lefschetz fixed-point theorem and the Riemann-Hurwitz formula. We recall them here:

Let $g: X \rightarrow X$ be a periodic, locally linear map on a compact manifold $X$. Then $\chi\left(X^{g}\right)=\lambda(g)$, where $\lambda(g)$, the Lefschetz number of $g$, is the alternating sum of $g$ 's traces on homology. (The formula holds more generally, but this suffices for our purposes.) In particular, if $g$ acts trivially on homology, $\chi\left(X^{g}\right)=\chi(X)$. In the context of homologically trivial pseudofree actions on $S^{2} \times S^{2}$, it follows that each element of $G$ apart from the identity has exactly four fixed points.

The Riemann-Hurwitz formula describes the orbit structure of a pseudofree action of a finite group $G$ on a compact space $X$. If the action has singular orbits $G x_{1}, \ldots, G x_{m} ;\left|G_{x_{i}}\right|=n_{i}$, and $|G|=N$, then

$$
\chi(X)=N \chi(X / G)-\sum_{i=1}^{m}\left(N-\frac{N}{n_{i}}\right) .
$$

Again, the theorem generalizes - this time, to nonpseudofree actions. See [Kulkarni 1982], for example. In our case $\left(X=S^{2} \times S^{2}\right.$; homologically trivial actions, for now), transfer considerations show that $\chi(X)=\chi(X / G)=4$. Thus

$$
N=\frac{4}{4-\sum_{i=1}^{m}\left(1-\frac{1}{n_{i}}\right)} .
$$

Definition. The Riemann-Hurwitz data for a pseudofree action is the $m$-tuple $\left(n_{1}, \ldots, n_{m}\right)$ described in the statement of the formula; each number $n_{i}$ is the size of the isotropy subgroup corresponding to one orbit.

In the case of pseudofree actions of a group $G$ on $S^{2}$, it is easily seen that $m \leq 3$, and the only possible Riemann-Hurwitz data are of the form $(N, N),(2,2, k)$, $(2,3,3),(2,3,4)$, or $(2,3,5)$. By local linearity, each isotropy group is cyclic, and then group theory calculations show that the only possible groups are cyclic, dihedral, tetrahedral, octahedral, or icosahedral (see [Kulkarni 1982] or Lemma 4.4 below). In contrast, in the case $X=S^{2} \times S^{2}$, an analogous calculation to the one in the $S^{2}$ case only gives the bound $m \leq 7$. There are some 20 or so infinite families of such $m$-tuples satisfying the Riemann-Hurwitz formula, plus a finite, but quite large, number of exceptional solutions. Also, the tuples only describe the sizes, and not the structures, of the isotropy groups. From this point of view, then, an argument directly analogous to the one in the $S^{2}$ case would be intractable. However, the linear examples exhibit two more salient features: 
(1) A priori, the isotropy groups might be any groups admitting free orthogonal actions on $S^{3}$ (under the tangent space representation). In fact, they are all cyclic.

(2) Each element of $G$ has the four fixed points $(x, y),(x,-y),(-x, y)$, and $(-x,-y)$. In the cyclic, tetrahedral, and icosahedral cases, all four fixed points lie in different orbits, while in the dihedral and octahedral cases, the fixed-point sets of some elements meet two orbits in two points each. But all four fixed points of $g \in G$ never lie in the same orbit. Thus in the list of isotropy groups corresponding to the Riemann-Hurwitz data, each isotropy group actually occurs two or four times. Because of this repetition, the data in this case correspond to the data for an action on $S^{2}$, where things are simpler.

Our strategy, then, is to show that in the general case,

(1) The isotropy groups are still cyclic.

(2) They still occur in pairs in the Riemann-Hurwitz data,

and then appeal to the proof in the $S^{2}$ case, to show:

Theorem 4.1. A group acting pseudofreely, locally linearly, and homologically trivially on $S^{2} \times S^{2}$ is polyhedral.

The proof will use a series of lemmas. We first describe the possible isotropy groups:

Proposition 4.2. Suppose a finite group $G$ acts pseudofreely, homologically trivially, and locally linearly on a closed, simply-connected four-manifold $X$ with $b_{2}(X) \geq 2$. Then each isotropy group is cyclic.

Proof. It is shown in [McCooey 2002] that, without the pseudofree assumption, such an isotropy group $G_{x_{0}}$ must be abelian of rank 1 or 2 . But a rank 2 group cannot act freely on the linking sphere to $x_{0}$, and thus cannot act pseudofreely on $X$.

Lemma 4.3. Let $G$ and $X$ be as in Proposition 4.2. For each singular point $x$, let $o\left(X^{G_{x}}\right)$ be the number of $G$-orbits which meet $X^{G_{x}}$. Then $o\left(X^{G_{x}}\right)$ divides $\chi(X)$.

Proof. Since $G_{x}=\langle g\rangle$ for some $g,\left|X^{G_{x}}\right|=|\operatorname{Fix}(g)|=\lambda(g)=\chi(X)$.

Observe that for any $h \in G$, if $h^{-1} g h(x)=x$, then $h(x) \in \operatorname{Fix}(g)$. In other words, $N_{G}(\langle g\rangle)$ acts on $\operatorname{Fix}(g)$. Two points of $\operatorname{Fix}(g)$ are in the same $G$-orbit if and only if they are in the same $N_{G}(\langle g\rangle)$-orbit, for if $g x=x, g y=y$, and $k x=y$, then $k^{-1} g k \in G_{x}=\langle g\rangle$. But each $N_{G}(\langle g\rangle)$-orbit of the action on $\operatorname{Fix}(g)$ has cardinality $\left|N_{G}(g) /\langle g\rangle\right|$. Since they all have the same size, the number of orbits must divide $\chi(X)$. 
Now, corresponding to the set of singular orbits (and hence to the RiemannHurwitz data $\left.\left(n_{1}, \ldots, n_{m}\right)\right)$, there is a list orbit types $G_{x_{1}}, \ldots, G_{x_{n}}$. We say the groups are repeated in pairs if each orbit type occurs in this list an even number of times.

Lemma 4.4. Let $G$ act homologically trivially and pseudofreely on $S^{2} \times S^{2}$. If the isotropy groups of the $G$-action are repeated in pairs, then $G$ is polyhedral.

Proof. Since the groups occur in pairs, $m$ is even. Let $m^{\prime}=m / 2$, and rearrange the list so that $G_{1}=G_{m^{\prime}+1}, G_{2}=G_{m^{\prime}+2}$, and so forth. Since $N\left(4-\sum_{i=1}^{m}\left(1-1 / n_{i}\right)\right)=$ 4 , it follows that $2=N\left(2-\sum_{i=1}^{m^{\prime}}\left(1-1 / n_{i}\right)\right)$. As in the $S^{2}$ case, the possible $n_{1}, \ldots, n_{m^{\prime}}$ are $(N, N),(2,2, k),(2,3,3),(2,3,4)$, and $(2,3,5)$. In each case, these numbers represent the sizes of maximal cyclic subgroups of $G$, and each conjugacy class of maximal cyclic subgroups occurs in the list. Kulkarni [1982] describes the groups that can correspond to this data. Proofs are omitted for some of his assertions, and some details are different in our case and his, so we repeat the argument here.

$(N, N)$ clearly corresponds to a cyclic group of order $N$.

For the remaining cases, we make the following observations: If a maximal cyclic subgroup $\langle g\rangle$ of $G$ is also normal, it has index $\leq 2$ in $G$. For $G /\langle g\rangle$ operates freely on $\operatorname{Fix}(g)$, a set of four points. But since orbits come in pairs, $\operatorname{Fix}(g)$ must meet 2 or $4 G /\langle g\rangle$-orbits, so $|G /\langle g\rangle|=2$ or 1 . By a similar argument, any maximal cyclic subgroup intersecting the center of $G$ nontrivially has index $\leq 2$.

$(2,2, k)$ corresponds to a group of order $2 k$. It has a cyclic, index 2 subgroup $\langle g\rangle$ of order $k$, which must be normal. Kulkarni assumes that his groups operate on a space with $\chi=2$, and uses this fact to prove that each $h \in G \backslash\langle g\rangle$ must have order 2. In our case, we use the observation above. Now assume $h \in G \backslash\langle g\rangle$ is fixed. Since each $\left(h g^{a}\right)$ has order $2, h g^{a} h g^{a}=1$, so $h g^{a} h^{-1}=\left(g^{a}\right)^{-1}$. It follows that $G$ is dihedral.

In the $(2,3,3)$ case, $|G|=12 . \mathrm{G}$ is nonabelian. There are three nonabelian groups of order 12, and two of them contain elements of order 6 . The third is Tet.

In the $(2,3,4)$ case, $G$ has order 24 and trivial center. With this in mind, a look at a table of groups such as [Coxeter and Moser 1980, p. 137] easily shows that $G=$ Oct.

Finally, in the $(2,3,5)$ case, $|G|=60$. Sylow theory shows that $G$ contains five copies of $D_{2}$, intersecting trivially, 20 copies of $\mathbb{Z}_{3}$, and six copies of $\mathbb{Z}_{5}$. If any proper subgroup of $G$ were normal, it would contain every conjugate of each element. Counting arguments show this to be impossible, so $G$ is simple. Thus $G=$ Icos.

Lemma 4.5. Let $G$ act pseudofreely, locally linearly, and homologically trivially on $S^{2} \times S^{2}$. Then the isotropy groups of the $G$-action are repeated in pairs. 
Proof. Say $G$ is nice if for each singular point $x \in S^{2} \times S^{2},\left(S^{2} \times S^{2}\right)^{G_{x}}$ meets 2 or $4 G$-orbits. If $G$ is nice, then its isotropy groups are repeated in pairs. We assume inductively that every proper subgroup of $G$ is nice, but that $G$ is not. Then by Lemma 4.3, there is some $x_{0}$ so that $o\left(\left(S^{2} \times S^{2}\right)^{G_{x_{0}}}\right)=1$; that is, $G$ acts transitively on $\left.S^{2} \times S^{2}\right)^{G_{x_{0}}}$.

By Proposition 4.2, $G_{x_{0}}$ is cyclic - say $G_{x_{0}}=\langle g\rangle$, and Fix $(g)=\left\{x_{0}, \ldots, x_{3}\right\}$. Let $h_{1}\left(x_{0}\right)=x_{1}, h_{2}\left(x_{0}\right)=x_{2}$, and $h_{3}\left(x_{0}\right)=x_{3}$. By minimality, $G$ is generated by $g, h_{1}, h_{2}$, and $h_{3}$, and since $h_{i}^{-1} g h_{i}\left(x_{0}\right)=x_{0},\langle g\rangle$ is normal in $G$. By minimality again, $|g|=p$ for some prime $p$. Now, $G /\langle g\rangle$ acts freely on $\left\{x_{0}, \ldots, x_{3}\right\}$, so $|G /\langle g\rangle|=4$. Thus $G$ is an extension of $G_{x_{0}}$ by $\mathbb{Z}_{4}$ or $\mathbb{Z}_{2} \times \mathbb{Z}_{2}$.

The remainder of the proof is an analysis of these extensions. Most can be ruled out by elementary group theory considerations. The two more difficult cases use arguments essentially due to Edmonds [1997b].

In the following cases, consideration of the possible automorphism actions of $H$ on $\langle g\rangle$ shows that some element of $G \backslash\langle g\rangle$ must be central, and then that $g$ is contained in a cyclic subgroup of order $2 p$, contradicting minimality.

(1) $\mathbb{Z}_{p} \rtimes\left(\mathbb{Z}_{2} \times \mathbb{Z}_{2}\right)$, for $p>2$.

(2) Any nonsplit extension of $\mathbb{Z}_{2}$ by $\mathbb{Z}_{2} \times \mathbb{Z}_{2}$.

(3) $\mathbb{Z}_{p} \rtimes \mathbb{Z}_{4}$, for $p \equiv 3(\bmod 4)$.

The case $G=\mathbb{Z}_{2} \rtimes \mathbb{Z}_{4}$ must actually be abelian, so $G$ is a direct product. $\mathbb{Z}_{2} \times \mathbb{Z}_{4}$ contains two cyclic subgroups of order 4 . They intersect nontrivially, and therefore have the same fixed set. It follows that $G$ must act semifreely, that is, each singular point is fixed by the entire group.

Two cases remain: $G=\mathbb{Z}_{2} \times \mathbb{Z}_{2} \times \mathbb{Z}_{2}$, and $G=\mathbb{Z}_{p} \rtimes \mathbb{Z}_{4}$, where $p \equiv 1(\bmod 4)$.

Suppose $G=\mathbb{Z}_{2} \times \mathbb{Z}_{2} \times \mathbb{Z}_{2}$ admits a nonnice action. $G$ has seven cyclic subgroups. Since $\mathbb{Z}_{2} \times \mathbb{Z}_{2}$ does not act freely on $S^{3}$, their fixed-point sets are disjoint, and each has $\mathbb{Z}_{2}$ stabilizer. Since $G$ is abelian, it acts on each fixed-point set, so each constitutes an orbit. The action has Riemann-Hurwitz data (2, 2, 2, 2, 2, 2, 2).

Let $X$ be $S^{2} \times S^{2}$ minus a small invariant neighborhood of the singular set, and let $Y=X / G . Y$ is a compact 4-manifold with seven $\mathbb{R} P^{3}$ boundary components $P_{1}, \ldots, P_{7}$. The cohomology long exact sequence for the pair $(Y, \partial Y)$ (with $\mathbb{Z}_{2^{-}}$ coefficients) shows that $\operatorname{im}\left(i^{*}: H^{3}(Y) \rightarrow H^{3}(\partial Y)\right)$ has rank 6 . The covering $X \rightarrow Y$ is classified by a map $\varphi: Y \rightarrow B G$. This induces a map $(\varphi \circ i)^{*}: H^{3}(G) \rightarrow$ $H^{3}\left(\bigcup_{j} P_{j}\right)=\left(\mathbb{Z}_{2}\right)^{7}$, which factors through $H^{3}(Y)$. Since it factors, the rank of $\operatorname{im}(\varphi \circ i)^{*}$ is at most 6 .

On the other hand, each $\pi_{1}\left(P_{j}\right)$ maps to a different subgroup of $\pi_{1}(Y) \approx \mathbb{Z}_{2} \times$ $\mathbb{Z}_{2} \times \mathbb{Z}_{2}$ under the natural inclusion, and hence each $P_{j}$ corresponds to a different nontrivial element of $H_{1}(Y)$. Since $H_{1}(Y) \cong \operatorname{hom}\left(H_{1}(Y), \mathbb{Z}_{2}\right)$, each nontrivial element of $H^{1}\left(Y, \mathbb{Z}_{2}\right)$ restricts nontrivially to $H^{1}\left(P_{j}\right)$ for some $j$. By the Kunneth 
theorem and the cohomology structure of $H^{*}\left(\mathbb{R} P^{3}\right)$, each of these has a nonzero cube which maps to the top class of $P_{j}$. Thus $\operatorname{rk}\left(\operatorname{im}(\varphi \circ i)^{*}\right)=7$, a contradiction.

A somewhat similar argument covers the remaining case. Let $G \cong \mathbb{Z}_{p} \rtimes \mathbb{Z}_{4}$, with $p \equiv 1(\bmod 4)$. The semidirect product automorphism must have order 4; otherwise $G$ contains a cyclic subgroup $\mathbb{Z}_{2 p}$. Thus $G \approx\langle g, h| g^{p}=h^{4}=1, h^{-1} g h=$ $\left.g^{a}\right\rangle$, where $4 a=p+1$. $G$ has $p$ different subgroups of order 4 , all of which are conjugate, so if an action exists, it has Riemann-Hurwitz data $(4,4,4,4, p)$. Define $X$ and $Y$ as before. Then $Y$ has boundary consisting of five lens spaces $L_{4}, L_{4}, L_{4}, L_{4}$, and $L_{p}$, with associated inclusions $i_{1}, \ldots, i_{5}: L_{n_{j}} \hookrightarrow Y$. Once again, the covering $X \rightarrow Y$ is classified by a map $\varphi: Y \rightarrow B G$, with induced maps $\varphi \circ i_{j}: L_{n_{j}} \rightarrow B G$. However, the cohomology calculation is just a bit subtler this time.

For any coefficient module $M$, the transfer map gives an isomorphism

$$
H^{*}(G ; M) \rightarrow H^{*}\left(\mathbb{Z}_{p} ; M\right)^{\mathbb{Z}_{4}}
$$

With $\mathbb{Z}_{p}$ coefficients, the ring $H^{*}\left(\mathbb{Z}_{p}\right)$ is generated by elements $s \in H^{1}\left(\mathbb{Z}_{p}\right)$ and $t \in H^{2}\left(\mathbb{Z}_{p}\right)$, where $t$ is the image of $s$ under the Bockstein map. $H^{*}\left(\mathbb{Z}_{p}\right)$ therefore inherits a $G$-module structure from the action of $G$ on $s$ given by $h \cdot s=a s$. Thus $h \cdot t=a t$, and $h \cdot s t=(2 a) s t$, so the action of $h$ on $H^{3}\left(\mathbb{Z}_{p}\right)$ is given by multiplication by -1 . This has the unfortunate consequence that $H^{3}\left(G ; \mathbb{Z}_{p}\right)=0$. To compensate for the $G$-action on $H^{*}\left(\mathbb{Z}_{p}\right)$, we replace $\mathbb{Z}_{p}$ with a twisted coefficient module $\widetilde{\mathbb{Z}_{p}}$, where $h$ acts by -1 . Note that $H^{*}\left(\mathbb{Z}_{p} ; \widetilde{\mathbb{Z}_{p}}\right) \cong H^{*}\left(\mathbb{Z}_{p} ; \mathbb{Z}_{p}\right) \cong \mathbb{Z}_{p}$ as $\mathbb{Z}_{p}$-modules, since the restriction of the $G$-action to its subgroup $\mathbb{Z}_{p}$ is trivial. With this twisting, we observe:

(1) Restriction gives an isomorphism $\left(\varphi \circ i_{5}\right)^{*}: H^{3}\left(G ; \widetilde{\mathbb{Z}_{p}}\right) \rightarrow H^{3}\left(\mathbb{Z}_{p} ; \widetilde{\mathbb{Z}_{p}}\right) \cong \mathbb{Z}_{p}$.

(2) For $j=1, \ldots, 4$, the maps $\left(\varphi \circ i_{j}\right)^{*}: H^{3}\left(G ; \widetilde{\mathbb{Z}_{p}}\right) \rightarrow H^{3}\left(\mathbb{Z}_{4} ; \widetilde{\mathbb{Z}_{p}}\right)=0$ are trivial.

Now, since the coboundary map $\delta^{*}: H^{3}\left(\partial Y ; \widetilde{\mathbb{Z}_{p}}\right) \rightarrow H^{4}\left(Y, \partial Y ; \widetilde{\mathbb{Z}_{p}}\right)$ is Poincaré dual to the augmentation $H_{0}\left(\partial Y ; \widetilde{\mathbb{Z}_{p}}\right) \rightarrow H_{0}\left(Y ; \widetilde{\mathbb{Z}}_{p}\right)$, we see that

$$
\operatorname{im}\left(i^{*}: H^{3}\left(Y ; \widetilde{\mathbb{Z}_{p}}\right) \rightarrow H^{3}\left(\partial Y ; \widetilde{\mathbb{Z}_{p}}\right)\right)
$$

consists of all $\left(u_{1}, \ldots, u_{5}\right)$ in $H^{3}\left(\partial Y ; \widetilde{\mathbb{Z}_{p}}\right)$ such that $\sum u_{j}=0$. In particular, if $u_{1}+u_{2}+u_{3}+u_{4}=0$, then $u_{5}=0$, as well. But by the observations above, there are elements $u \in H^{3}\left(Y, \widetilde{\mathbb{Z}_{p}}\right)$ which restrict trivially to each $H^{3}\left(L_{4} ; \widetilde{\mathbb{Z}_{p}}\right)$, but nontrivially to $H^{3}\left(L_{p}, \widetilde{\mathbb{Z}_{p}}\right)$. This rules out the $G$-actions in question.

Theorem 4.1 follows from the lemmas. Thus condition 1 in Corollary 3.10 is necessary in the general case. In other words, any group acting pseudofreely and homologically trivially on $S^{2} \times S^{2}$ also admits a linear, homologically trivial, pseudofree action. We now proceed to prove the necessity of condition 2 . 
Theorem 4.6. Let $G$ act pseudofreely on $S^{2} \times S^{2}$, and suppose $\varphi(G)=\left\langle\left(\begin{array}{rr}-1 & 0 \\ 0 & -1\end{array}\right)\right\rangle$. Then $G$ is abelian, and $\operatorname{ker} \varphi$ is cyclic.

Proof. Let $K \subset G$ act homologically trivially, so that

$$
1 \rightarrow K \rightarrow G \stackrel{\varphi}{\rightarrow}\left\langle\left(\begin{array}{rr}
-1 & 0 \\
0 & -1
\end{array}\right)\right\rangle \approx \mathbb{Z}_{2} \rightarrow 1
$$

is exact.

Claim. $G / K$ acts freely on $S^{2} \times S^{2} / K$.

Let $u \in G \backslash K$, so that $\varphi(u)$ generates $\mathbb{Z}_{2}$. If, for some $x, u(x)$ lies in the same orbit as $x$, then for some $k \in K, k u(x)=x$. But $k u$ has Lefschetz number 0 , so if it has a fixed point, its fixed point set must be 2-dimensional.

For the same reason, $G / K$ acts freely on the set of singular points in $S^{2} \times S^{2} / K$, and therefore it identifies the paired orbits of Lemma 4.5. Choose a small $G$ invariant open neighborhood $N \subset S^{2} \times S^{2}$ of the singular set of the $K$-action, and let $X=S^{2} \times S^{2} \backslash N$. Let $Y=X / G$. It follows from the claim that $Y$ is a manifold with boundary consisting of two or three lens spaces $L_{n_{i}}$. (These $n_{i}$ 's are exactly those which appear in pairs in the Riemann- Hurwitz data.) Since $X$ is a cover of $Y$, we can use the Cartan-Leray spectral sequence $\left(E_{2}^{p, q} \cong H^{p}\left(G ; H^{q}(X)\right) \Rightarrow H^{p+q}(Y)\right)$ to compute $H^{2}(Y ; \mathbb{Z})$. Note that

(1) Since $G$ is finite, $H^{1}(G ; M)=0$ for any free $\mathbb{Z}$-module $M$.

(2) In general, $H^{0}(G ; M) \cong M^{G}$, the submodule of $M$ fixed by $G$. In our case, $G$ acts by $\left(\begin{array}{rr}-1 & 0 \\ 0 & -1\end{array}\right)$ on $H^{2}(X)$, so $H^{0}\left(G ; H^{2}(X)\right)=0$.

(3) No nonzero differentials enter or leave $E_{k}^{2,0} \cong H^{2}\left(G ; H^{0}(X)\right)$.

Thus $H^{2}(Y) \cong H^{2}(G)$. Similar arguments show that, for each component $L_{n_{i}}$ of $\partial Y, H^{2}\left(L_{n_{i}}\right) \cong H^{2}\left(\mathbb{Z}_{n_{i}}\right)$, and that the restriction $H^{2}(Y) \rightarrow H^{2}\left(L_{n_{i}}\right)$ is given by the corresponding map on subgroups.

By Poincaré duality in $\mathrm{Y}$, we have an exact sequence

$$
H^{2}(Y) \rightarrow H^{2}(\partial Y) \cong H_{1}(\partial Y) \rightarrow H_{1}(Y) .
$$

This becomes

$$
H^{2}(G) \stackrel{r_{\oplus}^{G}}{\rightarrow} \bigoplus H^{2}\left(\mathbb{Z}_{n_{i}}\right) \cong \bigoplus H_{1}\left(\mathbb{Z}_{n_{i}}\right) \stackrel{i_{\oplus}^{G}}{\rightarrow} H_{1}(G) .
$$

And since the restriction and inclusion maps factor through $K$, we also have a sequence

$$
H^{2}(K) \stackrel{r_{\oplus}^{K}}{\rightarrow} \bigoplus H^{2}\left(\mathbb{Z}_{n_{i}}\right) \cong \bigoplus H_{1}\left(\mathbb{Z}_{n_{i}}\right) \stackrel{i_{\oplus}^{K}}{\rightarrow} H_{1}(K) .
$$

This sequence is not exact in general. However, from the previous sequence, it follows that $H^{2}(\partial Y) / \operatorname{im}\left(r_{\oplus}^{G}\right)$ injects into $H_{1}(K)$, and that $H^{2}(\partial Y) / \operatorname{im}\left(r_{\oplus}^{K}\right)$ injects 
into a quotient of $H_{1}(K)$. The remainder of the proof consists of cohomology calculations showing that this is impossible unless $G$ is abelian and $K$ is cyclic.

Case 1: $K$ is cyclic. Since $G$ maps onto $\mathbb{Z}_{2},[G, G] \subseteq K$. Thus if $G$ is nonabelian, the kernel of $\langle k\rangle \rightarrow H_{1}(G)$ is nontrivial. In the exact sequence

$$
H^{2}(G) \stackrel{r_{\oplus}^{G}}{\rightarrow} H^{2}\left(\mathbb{Z}_{n}\right) \oplus H^{2}\left(\mathbb{Z}_{n}\right) \cong H_{1}\left(\mathbb{Z}_{n}\right) \oplus H_{1}\left(\mathbb{Z}_{n}\right) \stackrel{i_{\oplus}^{G}}{\rightarrow} H_{1}(G)
$$

the image of $r_{\oplus}^{G}$ lies in the diagonal subgroup of $H^{2}\left(\mathbb{Z}_{n}\right) \oplus H^{2}\left(\mathbb{Z}_{n}\right)$. If $G$ is nonabelian, the kernel of the inclusion map does not.

Case 2: $K=$ Tet, Oct, or Icos. To every polyhedral group $K$, there corresponds a binary polyhedral group $\widetilde{K}$ such that $1 \rightarrow \mathbb{Z}_{2} \rightarrow \widetilde{K} \rightarrow K \rightarrow 1$ is exact. The LyndonHochschild-Serre spectral sequence $\left(E_{2}^{p, q} \cong H^{p}\left(K ; H^{q}\left(\mathbb{Z}_{2}\right)\right) \Rightarrow H^{p+q}(\widetilde{K})\right.$, in this case) relates the cohomologies of the groups in this sequence. It shows, in particular, that $H^{2}(K)$ injects into $H^{2}(\widetilde{K})$. But since each $\widetilde{K}$ acts freely on $S^{3}$, Poincaré duality shows that $H^{2}(\widetilde{K}) \approx H_{1}(\widetilde{K})$. Thus $H^{2}(K) \hookrightarrow H_{1}(\widetilde{K})$.

By computing the sizes of $H_{1}(\widetilde{K})$ in each case, we find that $\left|H^{2}(\mathrm{Tet})\right| \leq 3$, $\mid H^{2}$ (Oct) $\mid \leq 2$, and $H^{2}(\mathrm{Icos})=0$. On the other hand, $\left|\bigoplus H^{2}\left(\mathbb{Z}_{n_{i}}\right)\right|=n_{1} \times$ $n_{2} \times n_{3}$. Finally, $H_{1}$ (Tet) $=\mathbb{Z}_{3}, H_{1}(\mathrm{Oct})=\mathbb{Z}_{2}$, and $H_{1}(\mathrm{Icos})=0$. In each case, $H^{2}(\partial Y) / \operatorname{im}\left(r_{\oplus}^{K}\right)$ is too large to inject into a quotient of $H_{1}(K)$.

Case 2: $K=D_{n}$. Recall that

$$
H_{1}\left(D_{n}\right) \approx\left\{\begin{array}{l}
\mathbb{Z}_{2}=\langle\bar{t}\rangle \text { if } n \text { is odd, } \\
\mathbb{Z}_{2} \times \mathbb{Z}_{2}=\langle\bar{s}, \bar{t}\rangle \text { if } n \text { is even, }
\end{array}\right.
$$

and

$$
H^{2}\left(D_{n}\right) \approx\left\{\begin{array}{l}
\mathbb{Z}_{2} \text { if } n \text { is odd, } \\
\mathbb{Z}_{2} \times \mathbb{Z}_{2} \text { if } n \text { is even. }
\end{array}\right.
$$

Since $n_{1}=n_{2}=2$ and $n_{3}=n$, we have $\left|\bigoplus H^{2}\left(\mathbb{Z}_{n_{i}}\right)\right|=4 n$. Except in the cases $n=2$ and $n=4$, it is immediate that $H^{2}(\partial Y) / \operatorname{im}\left(r_{\oplus}^{K}\right)$ cannot inject into any quotient of $H_{1}\left(D_{n}\right)$. We consider the remaining possibilities:

Suppose $n=2$. Using Lemma 2.2, we find that the only nonabelian extension $1 \rightarrow D_{2} \rightarrow G \rightarrow \mathbb{Z}_{2} \rightarrow 1$ has $G=D_{4} \cong\left\langle s, t \mid s^{4}=t^{2}=1, t s t^{-1}=s^{-1}\right\rangle$, with $D_{2}=\left\langle s^{2}, t\right\rangle$. Thus we have an exact sequence $H^{2}\left(D_{4}\right) \stackrel{r_{\oplus}^{D_{4}}}{\rightarrow} H^{2}\left(\mathbb{Z}_{2}\right) \oplus H^{2}\left(\mathbb{Z}_{2}\right) \oplus H^{2}\left(\mathbb{Z}_{2}\right) \cong H_{1}\left(\mathbb{Z}_{2}\right) \oplus H_{1}\left(\mathbb{Z}_{2}\right) \oplus H_{1}\left(\mathbb{Z}_{2}\right) \stackrel{i_{\oplus}^{D_{4}}}{\rightarrow} H_{1}\left(D_{4}\right)$,

where the three $\mathbb{Z}_{2}$ subgroups are generated by $s^{2}, t$, and $s^{2} t$. Since the restrictions and inclusions factor through $D_{2}$, Lemma 2.5 shows that $\left|\operatorname{im}\left(r_{\oplus}^{D_{4}}\right)\right|=\left|\operatorname{ker} i_{\oplus}^{D_{4}}\right|=2$. This contradicts exactness. 
$D_{2}$ also has two abelian extensions: $1 \rightarrow D_{2} \rightarrow D_{2} \times \mathbb{Z}_{2} \rightarrow \mathbb{Z}_{2} \rightarrow 1$, and $1 \rightarrow D_{2} \rightarrow \mathbb{Z}_{4} \times \mathbb{Z}_{2} \rightarrow \mathbb{Z}_{2} \rightarrow 1$. In each case, the restriction and inclusion maps can be explicitly calculated, and the sequence is seen not to be exact. $D_{2}$ is the only candidate for $\operatorname{ker} \varphi$ which is abelian, but not cyclic.

Finally, suppose $K=D_{4}$. Then we have $\mathbb{Z}_{n_{1}}=\langle t\rangle \approx \mathbb{Z}_{2}, \mathbb{Z}_{n_{2}}=\langle s t\rangle \approx \mathbb{Z}_{2}$, and $\mathbb{Z}_{n_{3}}=\langle s\rangle \approx \mathbb{Z}_{4}$. Consulting Lemma 2.5 again, we see that $r_{\oplus}^{D_{4}}$ has matrix $\left(\begin{array}{ll}0 & 1 \\ 1 & 0 \\ 2 & 2\end{array}\right)$ relative to the bases given there; $i_{\oplus}^{D_{4}}$ has matrix $\left(\begin{array}{lll}0 & 1 & 1 \\ 1 & 1 & 0\end{array}\right)$, as is easily checked.

Now, if $r_{D_{4}}^{G}$ is not onto, then the element counts which applied for most $n$ also apply for $D_{4}$. But if $r_{D_{4}}^{G}$ is onto, then $\bigoplus H^{2}\left(\mathbb{Z}_{n_{i}}\right) / \mathrm{im}\left(r_{\oplus}^{D_{4}}\right)$ should inject into $H_{1}\left(D_{4}\right)$, so we should have $\operatorname{ker} i_{\oplus}^{D_{4}} \subseteq \operatorname{im}\left(r_{\oplus}^{D_{4}}\right)$. However, the element $(1,1,1) \in$ $H_{1}(\langle t\rangle) \oplus H_{1}(\langle s t\rangle) \oplus H_{1}(\langle s\rangle)$ is in the kernel, but not in the image.

Condition 3 of Corollary 3.10 is necessary by Bredon's theorem. A related result of Bredon helps us establish the necessity of condition 4, and thus complete the proof of the main theorem:

Proposition 4.7. Suppose $G$ acts pseudofreely, $\varphi\left(q_{a}\right)=a, \varphi\left(q_{b}\right)=b$, and $q_{a}^{2}=1$. Then $q_{a}$ and $q_{b}$ do not commute.

Proof. Given the necessity of conditions 1, 2, and 3 of Corollary 3.10, it suffices to rule out the possibility that $G=\left\langle q_{a}, q_{b}\right\rangle \cong \mathbb{Z}_{2} \times \mathbb{Z}_{4 k}$, where $k \geq 1$. In the case of the linear models (Proposition 3.8), the argument divided into two parts: If $q_{a}=(\alpha, \alpha)$, then $q_{a} q_{b}^{2 k}$ fixes a torus, contradicting pseudofreeness. And if $q_{a}=(\alpha, \rho)$ or $(\rho, \alpha)$, then the fact that $q_{b}$ exchanges factors of $S^{2} \times S^{2}$ means that $q_{a}$ and $q_{b}$ cannot commute, so no action exists, even with a two-dimensional singular set.

Assume, then, that $q_{a}$ and $q_{b}$ commute. Now, $q_{a}$ must act freely, so $X=S^{2} \times$ $S^{2} /\left\langle q_{a}\right\rangle$ is a manifold which inherits an action by $\left\langle q_{b}\right\rangle=\mathbb{Z}_{4 k}$. As motivation, we note that the linear models for $q_{a}$ are distinguished by the intersection forms (with $\mathbb{Z}_{2}$ coefficients) of the quotient spaces: In $X_{1}=S^{2} \times S^{2} /(\alpha, \alpha)$, the diagonal $S^{2}$ maps to an embedded $\mathbb{R} P^{2}$ with self-intersection 1 , so $w_{2}\left(X_{1}\right) \neq 0$. On the other hand, generators of second homology for $X_{2}=S^{2} \times S^{2} /(\alpha, \rho)$ are given by the images of $S^{2} \times *$, (where $*$ is some point fixed by $\rho$ ), and $(* \cup-*) \times S^{2}$, (where $*$ is any point in the first factor). Each of these has trivial self-intersection, so $w_{2}\left(X_{2}\right)=0$. To complete the proof, we will show (just as in the linear models) that if $\left\langle q_{b}\right\rangle$ acts on $X$, then $w_{2}(X) \neq 0$. Bredon's theorem then guarantees a two-dimensional singular set.

Let $x$ and $y$ denote the standard generators for $H^{2}\left(S^{2} \times S^{2} ; \mathbb{Z}_{2}\right)$, and consider the cohomology spectral sequence of the covering:

$$
E_{2}^{p, q}(X)=H^{p}\left(\mathbb{Z}_{2} ; H^{q}\left(S^{2} \times S^{2} ; \mathbb{Z}_{2}\right)\right) \Rightarrow H^{p+q}\left(X ; \mathbb{Z}_{2}\right) .
$$




\begin{tabular}{c|ccccccc}
$\langle x y\rangle$ & $\mathbb{Z}_{2}$ & $\mathbb{Z}_{2}$ & $\mathbb{Z}_{2}$ & $\mathbb{Z}_{2}$ & $\mathbb{Z}_{2}$ & $\mathbb{Z}_{2}$ & $\cdots$ \\
$\langle x, y\rangle$ & 0 & 0 & 0 & 0 & 0 & 0 & \\
& $\mathbb{Z}_{2} \oplus \mathbb{Z}_{2}$ & $\mathbb{Z}_{2} \oplus \mathbb{Z}_{2}$ & $\mathbb{Z}_{2} \oplus \mathbb{Z}_{2}$ & $\mathbb{Z}_{2} \oplus \mathbb{Z}_{2}$ & $\mathbb{Z}_{2} \oplus \mathbb{Z}_{2}$ & $\mathbb{Z}_{2} \oplus \mathbb{Z}_{2}$ & $\cdots$ \\
$\langle 1\rangle$ & 0 & 0 & 0 & 0 & 0 & 0 & \\
\hline & $\mathbb{Z}_{2}$ & $\mathbb{Z}_{2}$ & $\mathbb{Z}_{2}$ & $\mathbb{Z}_{2}$ & $\mathbb{Z}_{2}$ & $\mathbb{Z}_{2}$ & $\cdots$ \\
\hline & $\mathbb{Z}_{2}$ & $\langle s\rangle$ & $\left\langle s^{2}\right\rangle$ & $\left\langle s^{3}\right\rangle$ & $\left\langle s^{4}\right\rangle$ & $\left\langle s^{5}\right\rangle$ & $\cdots$
\end{tabular}

Table 1. $E_{2}(X)$.

It follows from the multiplicative structure of the spectral sequence that the behavior of the entire $E_{2}$ page is determined by $d_{2}(x)$ and $d_{2}(y)$. At least one must be nonzero, since the sequence converges to the cohomology of a four-manifold. And since $\left\langle q_{b}\right\rangle$ acts on the quotient, the differentials must respect the induced action of $q_{b}$ on $H^{2}\left(S^{2} \times S^{2}\right)$. Hence $d_{2}(x)=d_{2}(y)=1 s^{3}$, and ker $d_{2}$ is generated by $x+y$. It is easy to check that $E_{3}=\cdots=E_{\infty}$, so $u=[x+y]$ is a permanent cocycle.

This spectral sequence is identified by a homotopy equivalence with that of the fibration $S^{2} \times S^{2} \rightarrow E \mathbb{Z}_{2} \times \mathbb{Z}_{2}\left(S^{2} \times S^{2}\right) \rightarrow B \mathbb{Z}_{2}$ (see [Hu 1959, IX.15]), and under this identification, the cocycles which live to $E_{\infty}^{0, *}$ are those in the image of $p^{*}: H^{*}(X) \rightarrow H^{*}\left(S^{2} \times S^{2}\right)$. Thus there is a class $u \in H^{2}\left(X ; \mathbb{Z}_{2}\right)$ which lifts to $x+y \in H^{2}\left(S^{2} \times S^{2} ; \mathbb{Z}_{2}\right)$.

We claim that $u \cup u \neq 0$. To see this from the homological point of view, let $C_{*}(X)$ denote the singular chain complex of $X$. Then $S^{2} \times S^{2}=\widetilde{X}$, and $C^{*}(\widetilde{X} ; \mathbb{Z}) \cong$ $C^{*}(X ; \mathbb{Z}) \otimes_{\mathbb{Z}} \mathbb{Z}\left[\mathbb{Z}_{2}\right]$. The covering projection induces $p^{*}: C^{*}(X) \rightarrow C^{*}(\tilde{X})$ via $c \mapsto c \otimes\left(1+q_{a}\right)$. Moreover, because $C_{*}(X)$ is a free $\mathbb{Z}$-module, there is a natural isomorphism $\mu: C^{*}\left(X ; \mathbb{Z}_{)} \otimes \mathbb{Z}_{2} \rightarrow C^{*}\left(X ; \mathbb{Z}_{2}\right)\right.$, so that $H_{*}\left(C^{*}(X) \otimes \mathbb{Z}_{2}\right)=H^{*}\left(X ; \mathbb{Z}_{2}\right)$.

Every class in $H^{2}\left(S^{2} \times S^{2} ; \mathbb{Z}_{2}\right)$ is integral. Thus we can choose a cochain $v \in C^{*}(X ; \mathbb{Z})$ so that $\delta\left(p^{*}(v)\right)=\delta\left(v \otimes\left(1+q_{a}\right)\right)=0$ in $C^{3}(\tilde{X} ; \mathbb{Z})$ and such that $v \otimes 1$ is a cocycle representing $u$ in $H^{2}\left(X ; \mathbb{Z}_{2}\right)$. (Note that $v$ itself need not be an integral cocycle. For related discussion, see [Acosta and Lawson 1997].) Then $\left[p^{*}(v) \otimes 1\right]=x+y \in H^{2}\left(\widetilde{X} ; \mathbb{Z}_{2}\right)$, and $\left[p^{*}(v)\right] \in H^{2}(\widetilde{X} ; \mathbb{Z})$ represents an integral lift of $x+y-$ say $(2 m+1) \hat{x}+(2 n+1) \hat{y}$. So $\left[p^{*}(v) \cup p^{*}(v)\right]=(8 m n+4 m+$ $4 n+2)(\hat{x} \cup \hat{y}) \equiv 2 \bmod 4$.

But $p^{*}(v) \cup p^{*}(v)$ is also an equivariant cochain and hence must be of the form $\gamma \otimes\left(1+q_{a}\right)$, where $\gamma \in C^{4}(X ; \mathbb{Z})$. So $[\gamma] \equiv 1 \bmod 2$, and $[\gamma \otimes 1]$ represents $u \cup u$. Thus $u \cup u \neq 0$, so $w_{2}(X) \neq 0$.

A heuristic geometrical argument is more direct: the Poincaré dual of $u$ can be represented by a surface $F$, and $\widetilde{F} \subset S^{2} \times S^{2}$ will represent an integral lift of 
$P D(x+y)$, so $\widetilde{F} \cdot \widetilde{F} \equiv 2(\bmod 4)$. The intersection points will be paired up by the $\left\langle q_{a}\right\rangle$-action, so $F \cdot F \equiv 1(\bmod 2)$, and $u \cup u \neq 0$.

Combining Corollary 3.10, Theorem 4.1, Theorem 4.6, Proposition 4.7, and Proposition 3.4, we have our main theorem:

Theorem 4.8. Any finite group which admits a locally linear, orientation preserving, pseudofree action on $S^{2} \times S^{2}$ also admits a linear, orientation preserving, pseudofree action.

In fact, the pairs (group, cohomology representation) are exactly those which occur in the linear case.

\section{References}

[Acosta and Lawson 1997] D. Acosta and T. Lawson, "Even non-spin manifolds, $\operatorname{spin}^{c}$ structures, and duality", Enseign. Math. (2) 43:1-2 (1997), 27-32. MR 98m:57033 Zbl 0889.57013

[Bauer and Wilczyński 1996] S. Bauer and D. M. Wilczyński, "On the topological classification of pseudofree group actions on 4-manifolds, II", K-Theory 10:5 (1996), 491-516. MR 97m:57017 Zbl 0864.57012

[Bredon 1972] G. E. Bredon, Introduction to compact transformation groups, Pure and Applied Mathematics 46, Academic Press, New York, 1972. MR 54 \#1265 Zbl 0246.57017

[Brown 1982] K. S. Brown, Cohomology of groups, Graduate Texts in Mathematics 87, Springer, New York, 1982. MR 83k:20002 Zbl 0584.20036

[Cappell and Shaneson 1979] S. E. Cappell and J. L. Shaneson, "Pseudofree actions, I", pp. 395447 in Algebraic topology (Århus, 1978), edited by J. L. Dupont and I. H. Madsen, Lecture Notes in Math. 763, Springer, Berlin, 1979. MR 81d:57034 Zbl 0416.57020

[Coxeter and Moser 1980] H. S. M. Coxeter and W. O. J. Moser, Generators and relations for discrete groups, 4th ed., Ergebnisse der Mathematik 14, Springer, Berlin, 1980. MR 81a:20001 Zbl 0422.20001

[Edmonds 1997a] A. L. Edmonds, "Homologically trivial group actions on 4-manifolds", Preprint, 1997. math.GT/9809055

[Edmonds 1997b] A. L. Edmonds, "Pseudofree actions on spheres", unpublished notes, 1997.

[Edmonds and Ewing 1992] A. L. Edmonds and J. H. Ewing, "Realizing forms and fixed point data in dimension four", Amer. J. Math. 114:5 (1992), 1103-1126. MR 93i:57047 Zbl 0766.57020

[Hambleton and Lee 1988] I. Hambleton and R. Lee, "Finite group actions on $\mathrm{P}^{2}(\mathbf{C})$ ", J. Algebra 116:1 (1988), 227-242. MR 89e:14044 Zbl 0659.20041

[Hu 1959] S.-t. Hu, Homotopy theory, Pure and Applied Mathematics 8, Academic Press, New York, 1959. MR 21 \#5186 Zbl 0088.38803

[Kulkarni 1982] R. S. Kulkarni, "Pseudofree actions and Hurwitz's 84( $g-1)$ theorem”, Math. Ann. 261:2 (1982), 209-226. MR 84e:57034 Zbl 0479.57023

[Mac Lane 1975] S. Mac Lane, Homology, Grundlehren der Math. Wissenschaften 114, Springer, Berlin, 1975. MR 96d:18001 Zbl 0328.18009

[McCooey 2002] M. P. McCooey, "Symmetry groups of four-manifolds", Topology 41:4 (2002), 835-851. MR 2003f:57070 Zbl 1022.57009 
[Pearson 1996] K. Pearson, "Integral cohomology and detection of w-basic 2-groups", Math. Comp. 65:213 (1996), 291-306. MR 96d:20056 Zbl 0848.20045

[Rotman 1995] J. J. Rotman, An introduction to the theory of groups, 4th ed., Graduate Texts in Mathematics 148, Springer, New York, 1995. MR 95m:20001 Zbl 0810.20001

[Wilczyński 1987] D. M. Wilczyński, "Group actions on the complex projective plane”, Trans. Amer. Math. Soc. 303:2 (1987), 707-731. MR 88j:57041 Zbl 0639.57022

[Wilczyński 1994] D. M. Wilczyński, "On the topological classification of pseudofree group actions on 4-manifolds, I”, Math. Z. 217:3 (1994), 335-366. MR 96f:57014 Zbl 0813.57030

[Wolf 1984] J. A. Wolf, Spaces of constant curvature, 5th ed., Publish or Perish, Wilmington, DE, 1984. MR 88k:53002

Received November 1, 2004.

\section{Michael P. MCCOOEY}

DEPARTMENT OF MATHEMATICS

Franklin AND MARShall College

LANCASTER, PA 17604-3003

UNITED STATES

mmccooey@member.ams.org

http://edisk.fandm.edu/michael.mccooey/ 